\title{
Data report: magnetic properties of unconsolidated deep-sea sediments from the North Atlantic, IODP Expedition 303 Sites U1302-U1304 and U1308'
}

\author{
Noriko Kawamura, ${ }^{2}$ Naoto Ishikawa, ${ }^{3}$ and Masayuki Torii ${ }^{4}$
}

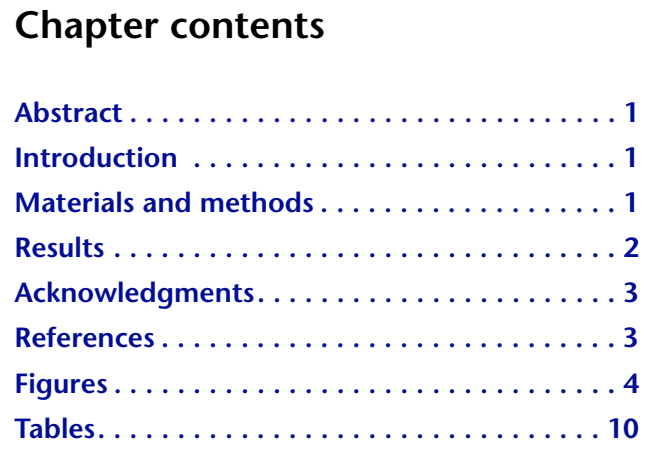

${ }^{1}$ Kawamura, N., Ishikawa, N., and Torii, M., 2009. Data report: magnetic properties of unconsolidated deep-sea sediments from the North Atlantic, IODP Expedition 303 Sites U1302U1304 and U1308. In Channell, J.E.T., Kanamatsu, T., Sato, T., Stein, R., Alvarez Zarikian, C.A. Malone, M.J., and the Expedition 303/306 Scientists, Proc. IODP, 303/306: College Station, TX (Integrated Ocean Drilling Program Management International, Inc.).

doi:10.2204/iodp.proc.303306.209.2009

${ }^{2}$ Japan Coast Guard Academy, 5-1 Wakaba-cho, Hiroshima 737-8512, Japan.

kawamura-noriko@jcga.ac.jp

${ }^{3}$ Graduate School of Human and Environmental Studies, Kyoto University, Yoshida-nihonmatsu-cho, Kyoto 606-8501, Japan.

${ }^{4}$ Department of Biosphere-Geosphere System Science, Okayama University of Science, Okayama 700-0005, Japan.

\begin{abstract}
Magnetic properties were measured on unconsolidated deep-sea sediments from Integrated Ocean Drilling Program Expedition 303 Sites U1302-U1304 and U1308 in the North Atlantic. Our results indicate general correspondences between rock magnetic parameters (i.e., remanent magnetization, low-field mass specific magnetic susceptibility, anhysteretic remanent magnetization, saturation remanent magnetization, and saturation magnetization) and visual core description-based lithology. Most magnetic grains in the sediments are in the pseudosingle-domain region, whereas magnetic grains in laminated diatom ooze at Site U1304 are in the multidomain region. Results of thermomagnetometry imply that the magnetic carrier in sediments at Sites U1302 and U1303 and at 20.9 meters composite depth (mcd) at Site U1308 is constituted by magnetite. On the other hand, maghemitized magnetite constitutes the magnetic carrier in sediments at Site U1304 and the upper 20.9 mcd of Site U1308.
\end{abstract}

\section{Introduction}

Rock magnetic properties of marine sediments vary with changes in the abundance, type, and grain size of magnetic minerals. In general, magnetic minerals in marine sediments are either detrital or authigenic in origin. Stability of iron oxides and sulfides in marine sediments is susceptible to changing redox conditions in interstitial waters with burial depth. Therefore, the original magnetic signals recorded in the sediments are subject to postdepositional diagenetic alteration that could complicate paleomagnetic and paleoenvironmental interpretations (e.g., Karlin and Levi, 1983).

To test the possible effect of early diagenesis on magnetic signals in the sediments, we qualitatively identified magnetic mineral species by measuring magnetic properties in hemipelagic sediments recovered from Integrated Ocean Drilling Program (IODP) Expedition 303 Sites U1302-U1304 and U1308 in the North Atlantic (Fig. F1).

\section{Materials and methods}

Sediment samples from Sites U1302-U1304 and U1308 used in this study were taken aboard the JOIDES Resolution during Expedi- 
tion 303 using either $7 \mathrm{~cm}^{3}$ plastic cubes or $1 \mathrm{~cm}^{3}$ plugs (i.e., splits of the shipboard carbon samples).

One cube was collected per section for the measurements. Wet cube samples were used to measure natural remanent magnetization (NRM), low-field massspecific magnetic susceptibility $(\chi)$, and anhysteretic remanent magnetization (ARM). NRM was measured with a 2G Model 760 superconducting rock magnetometer, and $\chi$ values were made with a Kappabridge KLY 3S at Kyoto University. Samples were given ARM in a steady direct current (DC) biasing field of $0.1 \mathrm{mT}$ in a peak alternating field (AF) of $80 \mathrm{mT}$ using a $2 \mathrm{G}$ Model 760 at the Geological Survey of Japan. ARM susceptibility $\left(\chi_{\text {ARM }}\right)$ is derived by dividing ARM by the bias magnetic field. NRM, $\chi$, and $\chi_{\text {ARM }}$ generally indicate the concentration of magnetic minerals in samples. Upon completion of above measurements, wet cube samples were then oven-dried at $40^{\circ} \mathrm{C}$ and weighed to calculate dry-based mass-specific magnetic parameters (Table T1).

Splits of the $1 \mathrm{~cm}^{3}$ plug samples (powdered) were used for magnetic hysteresis and thermomagnetic analyses. Two plug samples were collected per core (Table T2). Powder samples of $0.3-1 \mathrm{mg}$ were prepared for magnetic hysteresis analysis. Each powder sample was wrapped with aluminum foil $\sim 5 \mathrm{~mm}$ long. Coercivity (Hc), remanent coercivity (Hcr), saturation remanent magnetization $(\mathrm{Mr})$, saturation magnetization (Ms), and high-field magnetic susceptibility (HFMS) were obtained with an alternating gradient-force magnetometer (Princeton Measurement Corporation, MicroMag. Model 2900-02) at Kyoto University. Ms and Mr indicate the concentration of magnetic minerals in samples. Hcr and Hc are reflected in magnetic grain size in the samples. Using the definition by Day et al. (1977), Hcr/Hc and Mr/ Ms values are calculated and presented in Table $\mathbf{T} 2$. HFMS value was determined from the linear trend of the induced magnetization between 0.7 and $1.0 \mathrm{~T}$ and is influenced by paramagnetic, diamagnetic, and antiferromagnetic signals in samples. In order to diagnose for magnetic minerals, high- and lowtemperature magnetometry were performed. To measure low-temperature magnetometry, $\sim 20 \mathrm{mg}$ of powder sample was wrapped with plastic film. Isothermal remanent magnetization (IRM) was imparted at $5 \mathrm{~K}$ in a DC field of $1.0 \mathrm{~T}$, and changes in the IRM were measured after each heating step of $1 \mathrm{~K}$ up to $300 \mathrm{~K}$ using a Quantum Design MPMS XL5 at Okayama University of Science (samples from Sites U1302 and U1303) and the Center for Advanced Marine Core Research in Kochi University (samples from Sites U1304 and U1308). To measure hightemperature magnetometry, a sediment sample from Site U1308 was heated from room temperature to $600^{\circ} \mathrm{C}$ and cooled in air at a rate of $\sim 8^{\circ} \mathrm{C} / \mathrm{min}$ in an applied field of $0.4 \mathrm{~T}$ using a thermomagnetic balance at Doshisha University.

\section{Results Sites U1302 and U1303}

Downcore variations of rock magnetic parameters are shown in Figure F2. NRM variations show similar trends with $\chi, \chi_{\text {ARM }}, M r, M s$, and HFMS. Decreases of NRM, $\chi$, and $\chi_{\text {ARM }}$ values are found below 115 meters composite depth (mcd). The lithology changes from nannofossil silty clay to matrix-supported intraclast conglomerate downward (Tables T1, T2). Hcr/Hc and $\mathrm{Ms} / \mathrm{Mr}$ ratios suggest that most magnetic grains in the samples are in the pseudosingle-domain (PSD) region (Day et al., 1977). At 59 and $78-83$ mcd, a decrease in these ratios is found and lithology changes from clayey silt nannofossil ooze to slity clay (Table T2).

\section{Site U1304}

Rock magnetic parameters are shown in Figure F3. NRM, $\chi, \chi_{\text {ARM }}, M s$, and HFMS values fluctuate considerably and show similar downcore patterns. Extremely low values of NRM, $\chi$, and $\chi_{\text {ARM }}$ are observed at $46-47,57-58$, and $95-105 \mathrm{mcd}$. The $\mathrm{Hcr} / \mathrm{Hc}$ ratio is characterized by two maxima, and minima of $\mathrm{Mr} /$ Ms are recognized at these intervals. These values show that magnetic grains are possibly in the multidomain region. Except for these depths, Hcr/Hc and $\mathrm{Ms} / \mathrm{Mr}$ ratios indicate that magnetic grains in the samples are in the PSD region (Day et al., 1977). The laminated diatom ooze is recognized in these intervals (Tables T1, T2).

\section{Site U1308}

Downcore rock magnetic parameters are shown in Figure F4. NRM shows that relatively high values are found between 140 and 145 mcd. However, no notable change is observed in lithology at that depth interval (Tables T1, T2). The value of $\chi$ gradually increases downhole and is similar in overall trends to $\chi_{\text {ARM }}$ and Mr. The lithology gradually changes from nannofossil ooze silty clay to nannofossil ooze with depth (Tables T1, T2). Hcr/Hc and Mr/Ms values show that most magnetic grains in the samples are in the PSD region (Day et al., 1977).

\section{Thermomagnetometry}

Low-temperature magnetometry results are shown in Figure F5. Samples were mainly selected from the upper sediments. The Verwey transition suggesting the 
presence of magnetite (110-120 K) (Verwey, 1939) is clear in Site U1302 and U1303 samples (Fig. F5AF5E). Low-temperature magnetometry results from Site U1304 sediments are shown in Figure F5F-F5I. We can observe the unclear Verwey transition, which is indicative of the occurrence of maghemitization (Özdermir et al., 1993).

The thermomagnetic curve of the topmost sediments from Site U1308 is shown in Figure F6. The Curie point of magnetite $\left(\right.$ at $580^{\circ} \mathrm{C}$ ) is not clearly defined in the heating curve; it only appears on the cooling curve. In low-temperature magnetometry results for Site U1308 samples, the shape of the Verwey transition of Figure F5J-F5M is much more pronounced compared to that in Figure F5N. Verwey transitions are invisible above $7.5 \mathrm{mcd}$ (Fig. F5JF5K), but they are slightly evident below $11.0 \mathrm{mcd}$ (Fig. F5L-F5N).

\section{Acknowledgments}

We are grateful to Toshitsugu Yamazaki at the Geological Survey of Japan, Kazuto Kodama at Kochi University, and Koji Fukuma at Doshisya University for access to their facilities. We thank Joseph Stoner at Oregon State University and Atsuhito Ennyu at Itochu Oil Exploration for valuable comments that helped to improve our manuscript. This research used samples and/or data provided by the Integrated Ocean Drilling Program (IODP). Funding for this research was partially provided to N. Kawamura by the Fukada Geological Institute (Grant-in-Aid Program for Young Researchers Grant Number 15), the Minis- try of Education, Culture, Sports, Science and Technology (Scientific Research C Grant Number 19612002), and Paleo Lab (Grant-in-Aid Program for Young Researchers). Travel fund was provided to N. Kawamura by the Japan Agency for Marine-Earth Science and Technology (JAMSTEC) to participate in Expedition 303 in March-May 2004 and the second postcruise meeting in May 2007. A part of this study was performed under the cooperative research program of the Center for Advanced Marine Core Research, Kochi University (Numbers 05A003, 05B007).

\section{References}

Day, R., Fuller, M., and Schmidt, V.A., 1977. Hysteresis properties of titanomagnetites: grain-size and compositional dependence. Phys. Earth Planet. Inter., 13(4):260267. doi:10.1016/0031-9201(77)90108-X

Karlin, R., and Levi, S., 1983. Diagenesis of magnetic minerals in recent hemipelagic sediments. Nature (London, U. K.), 303(5915):327-330. doi:10.1038/303327a0

Özdemir, Ö., Dunlop, D.J., and Moskowitz, B.M., 1993. The effect of oxydation on the Verwey transition in magnetite. Geophys. Res. Lett., 20(16):1671-1674. doi:10.1029/93GL01483

Verwey, E.J.W., 1939. Electron conduction of magnetite $\left(\mathrm{Fe}_{3} \mathrm{O}_{4}\right)$ and its transition point at low temperatures. Nature (London, U. K.), 144(3642):327-328. doi:10.1038/144327b0

Initial receipt: 28 August 2008

Acceptance: 26 April 2009

Publication: 17 July 2009

MS 303306-209 
Figure F1. Location map of Expedition 303 sites.

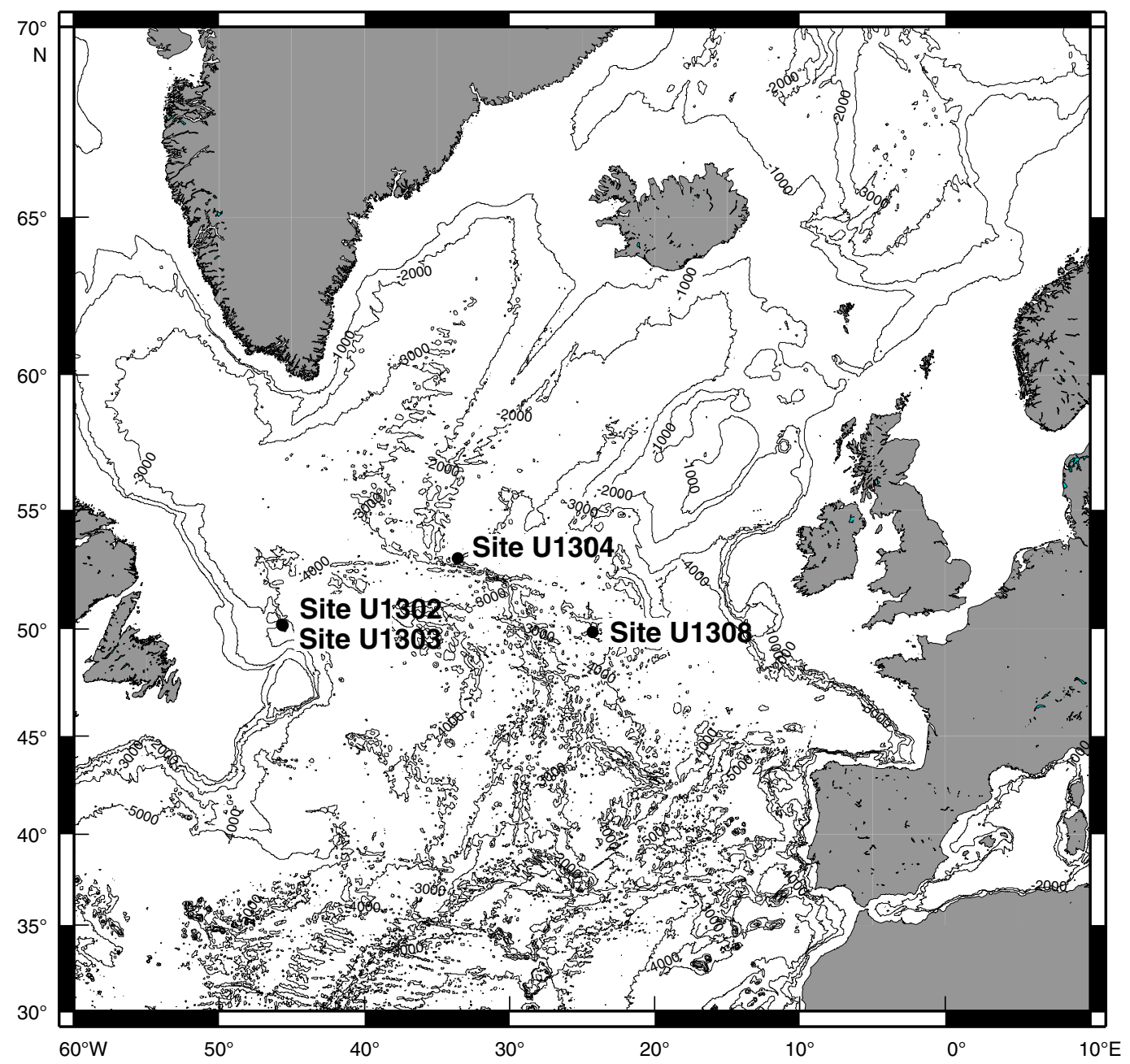


Figure F2. Rock magnetic parameters, Sites U1302 and U1303. NRM = natural remanent magnetization, $\chi=$ low-field magnetic susceptibility, $\chi_{\text {ARM }}$ = susceptibility of anhysteretic remanent magnetization (ARM), HFMS = high-field magnetic susceptibility, $\mathrm{Mr}=$ isothermal remanent magnetization, $\mathrm{Ms}=$ saturated magnetization, $\mathrm{Hc}=$ coercivity, $\mathrm{Hcr}=$ remanent coercivity. mcd = meters composite depth.

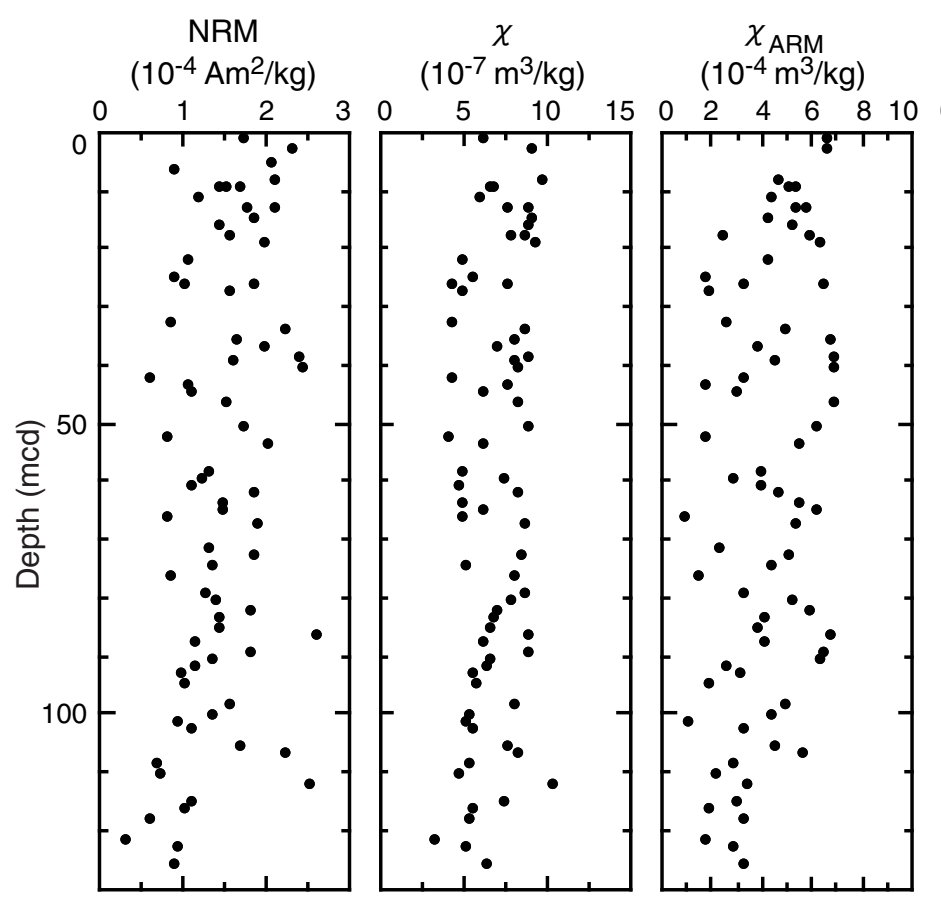

HFMS Magnetization

$\left(10^{-8} \mathrm{~m}^{3} / \mathrm{kg}\right)$

$\left(\mathrm{Am}^{2} / \mathrm{kg}\right)$

\begin{tabular}{lll}
$0.05 \quad 0.1$ & 0.15 \\
\hline
\end{tabular}
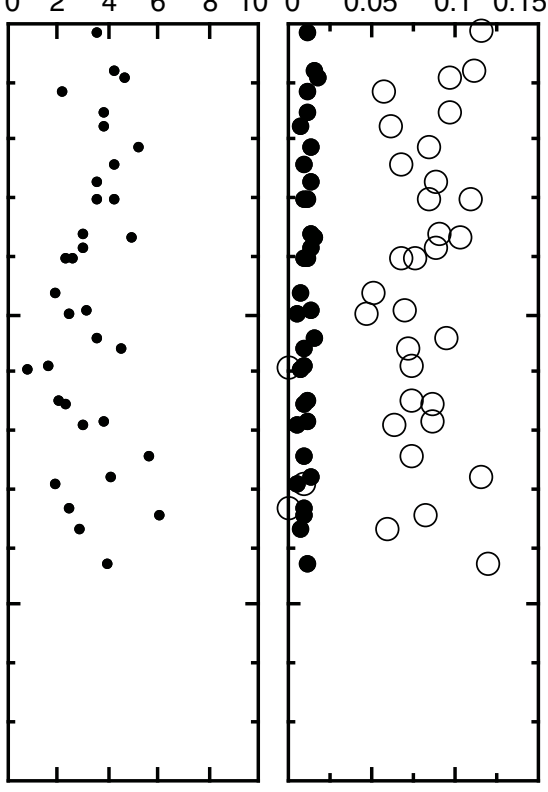

$\mathrm{Mr} / \mathrm{Ms}$

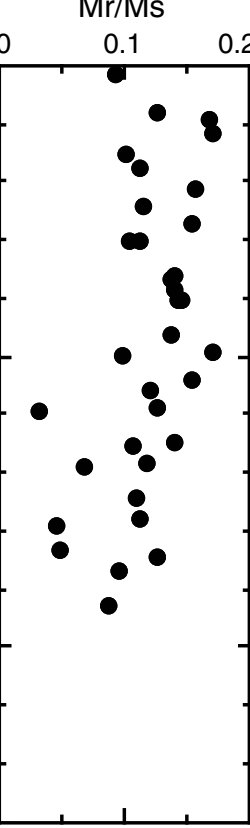

Coercivity
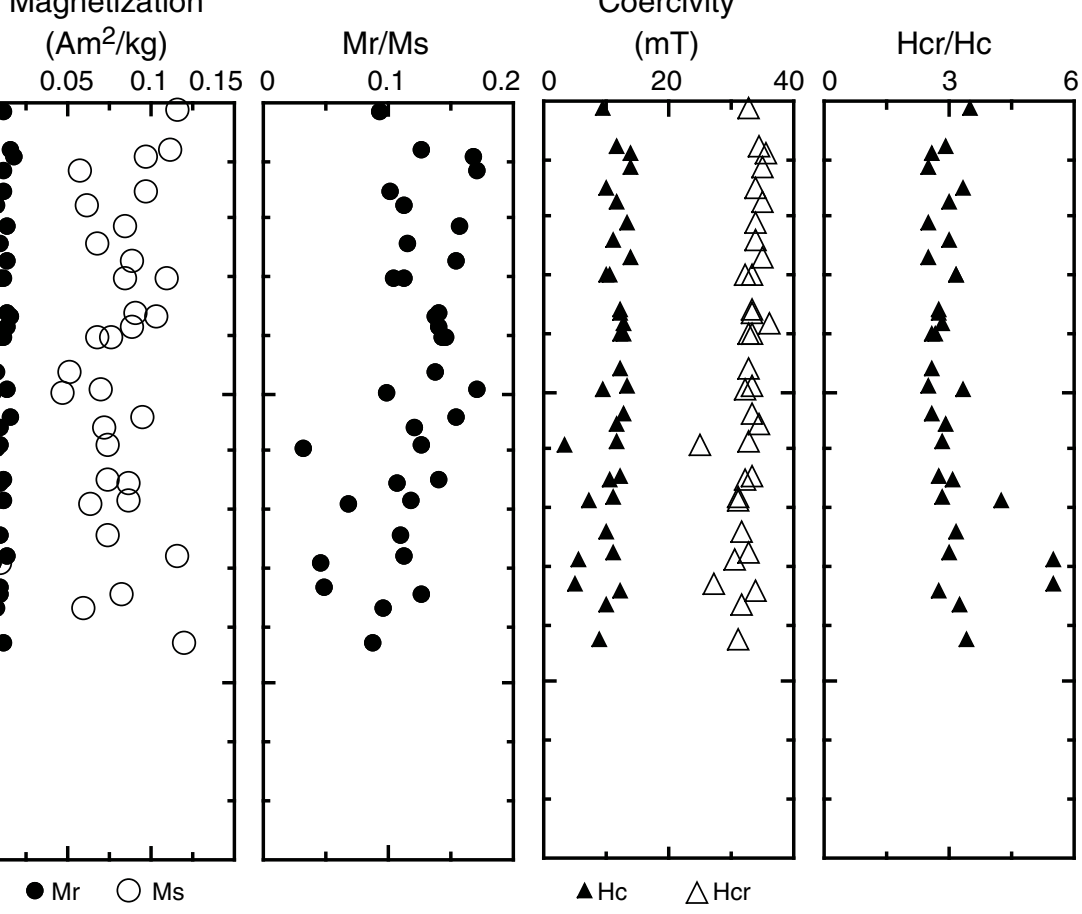

$\triangle \mathrm{Hcr}$ 
Figure F3. Rock magnetic parameters, Site U1304. NRM = natural remanent magnetization, $\chi=$ low-field magnetic susceptibility, $\chi_{\text {ARM }}=$ susceptibility of anhysteretic remanent magnetization (ARM), HFMS = high-field magnetic susceptibility, $\mathrm{Mr}=$ isothermal remanent magnetization, Ms = saturated magnetization, $\mathrm{Hc}=$ coercivity, $\mathrm{Hcr}=$ remanent coercivity. mcd = meters composite depth.

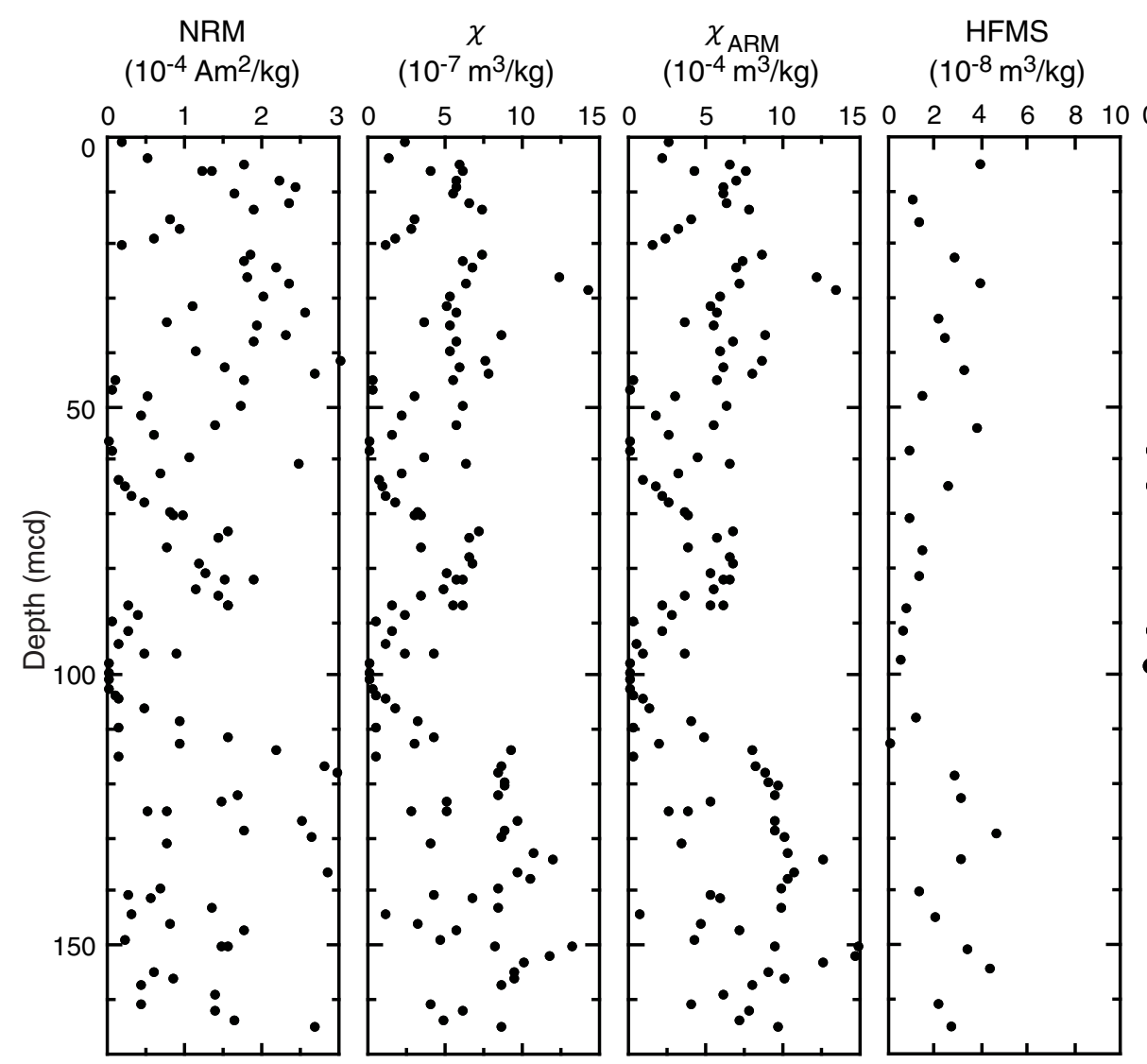

Magnetization

$\left(\mathrm{Am}^{2} / \mathrm{kg}\right)$

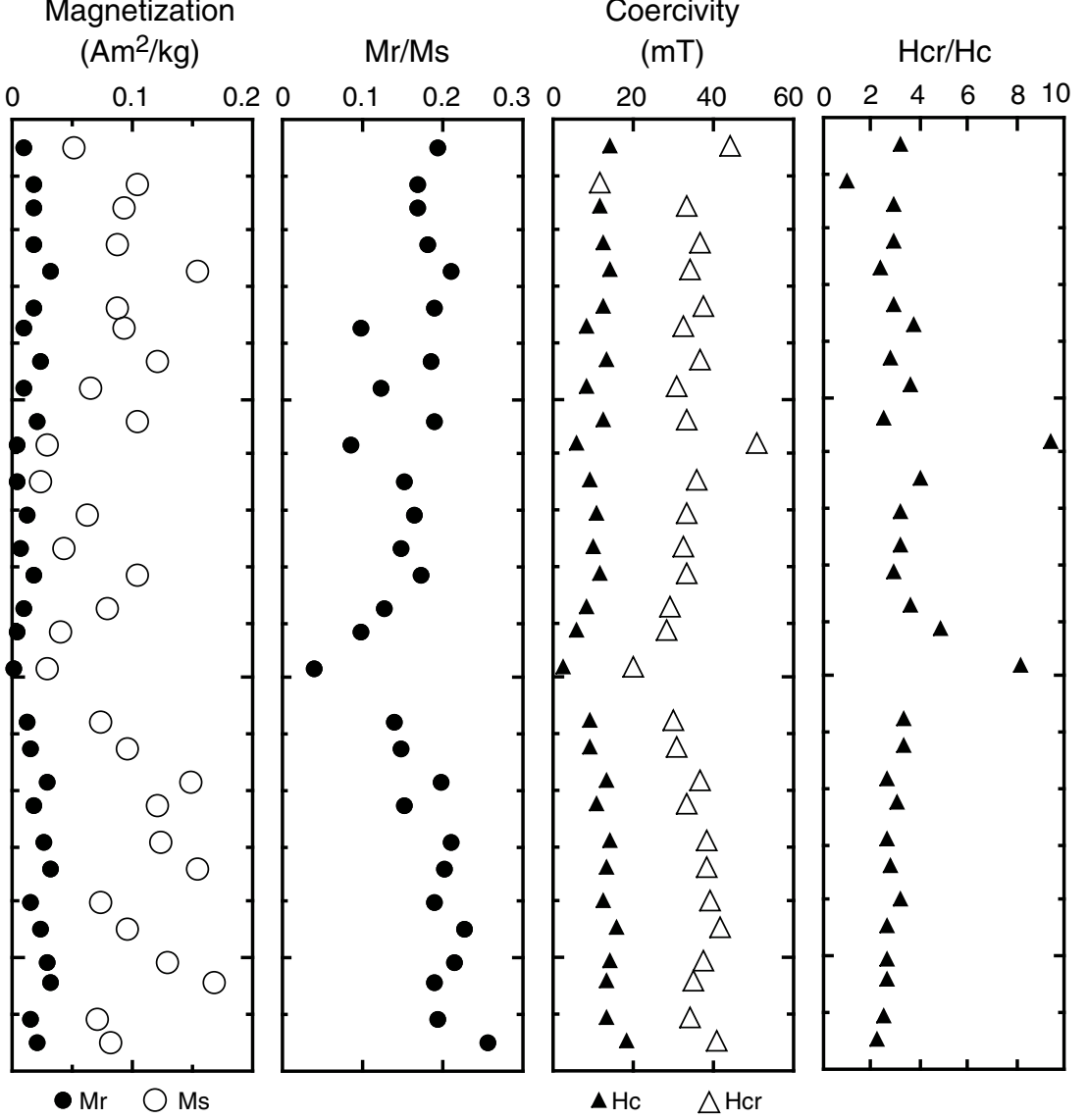

Coercivity

$(\mathrm{mT})$

$\mathrm{Hcr} / \mathrm{Hc}$ 
Figure F4. Rock magnetic parameters, Site U1308. NRM = natural remanent magnetization, $\chi=$ low-field magnetic susceptibility, $\chi_{\text {ARM }}=$ susceptibility of anhysteretic remanent magnetization (ARM), HFMS = high-field magnetic susceptibility, $\mathrm{Mr}=$ isothermal remanent magnetization, Ms = saturated magnetization, $\mathrm{Hc}=$ coercivity, $\mathrm{Hcr}=$ remanent coercivity. $\mathrm{mcd}=$ meters composite depth.

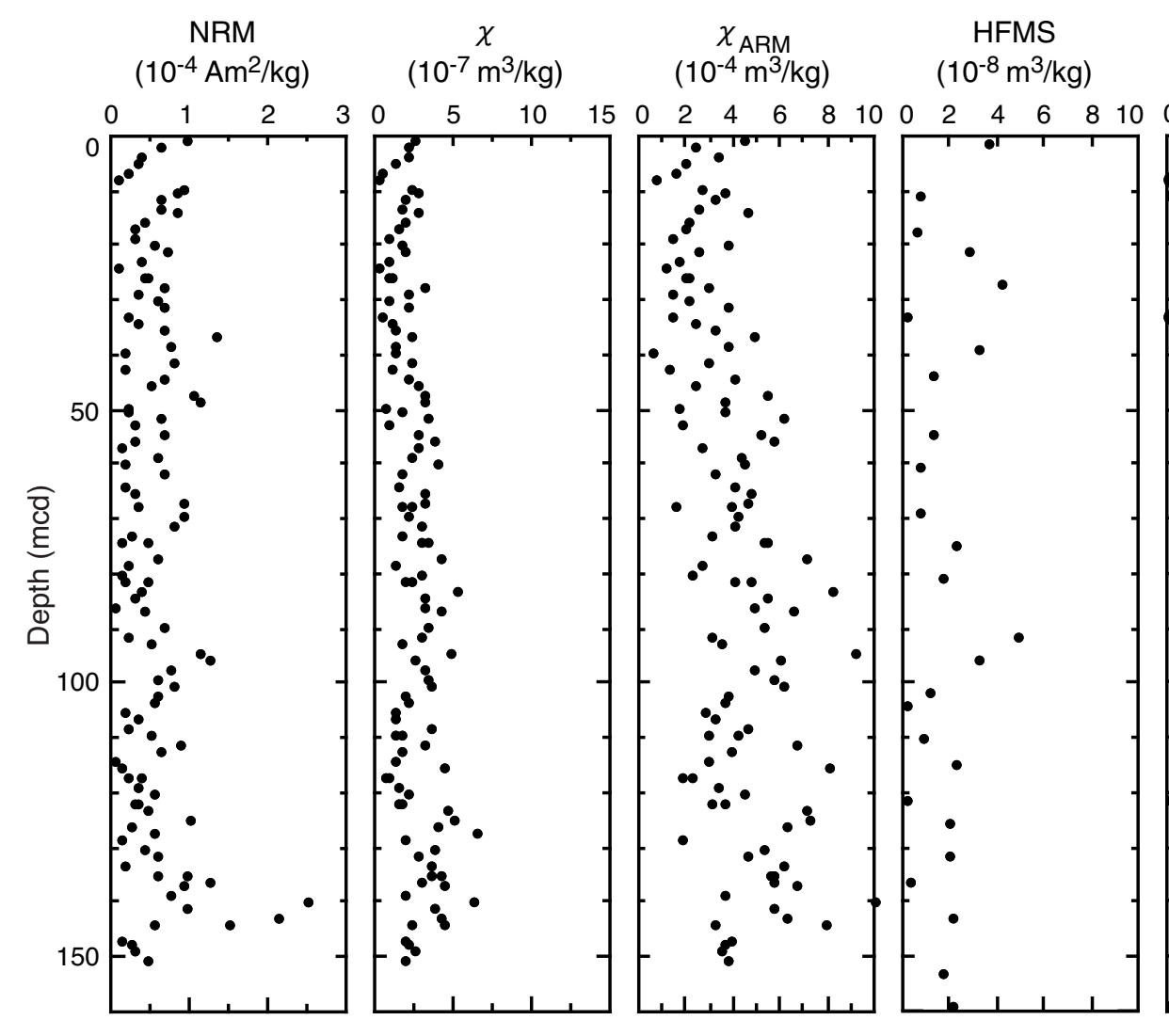

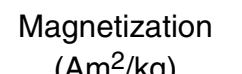

$\left(\mathrm{Am}^{2} / \mathrm{kg}\right)$

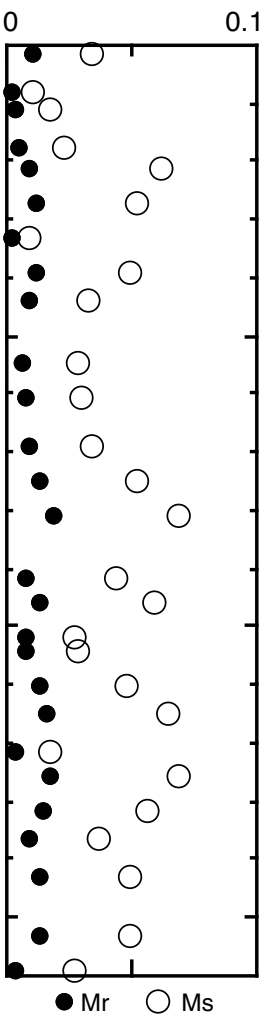

Coercivity

$(\mathrm{mT})$

$\mathrm{Hcr} / \mathrm{Hc}$

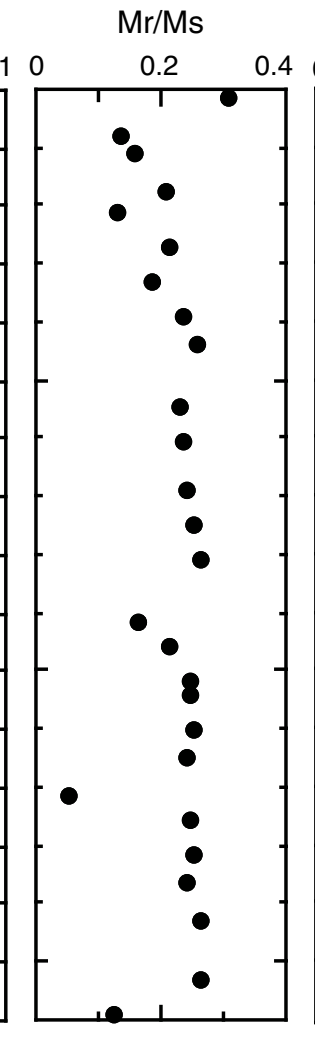

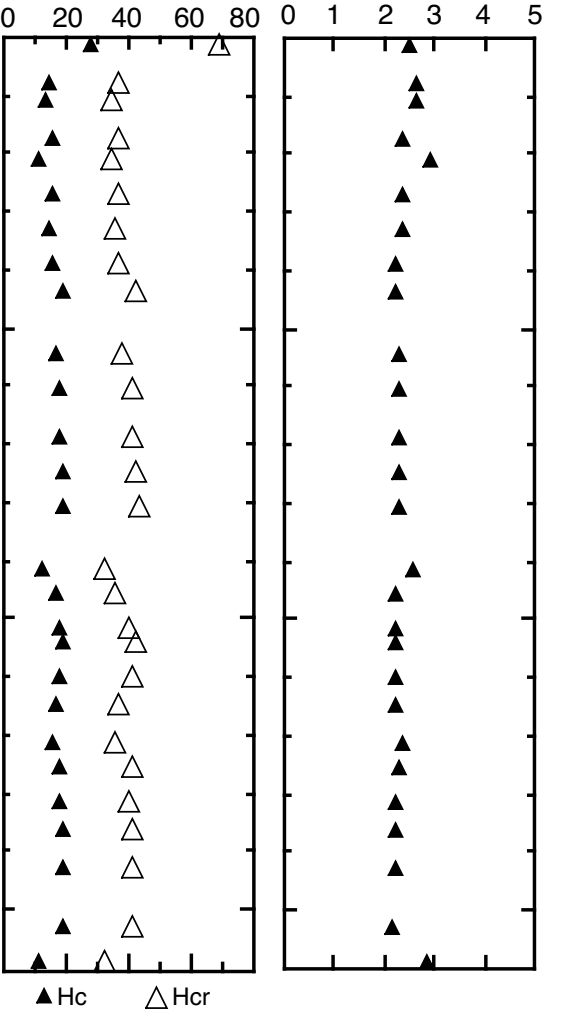


Figure F5. Results of low-temperature magnetometry. IRM = isothermal remanent magnetization.
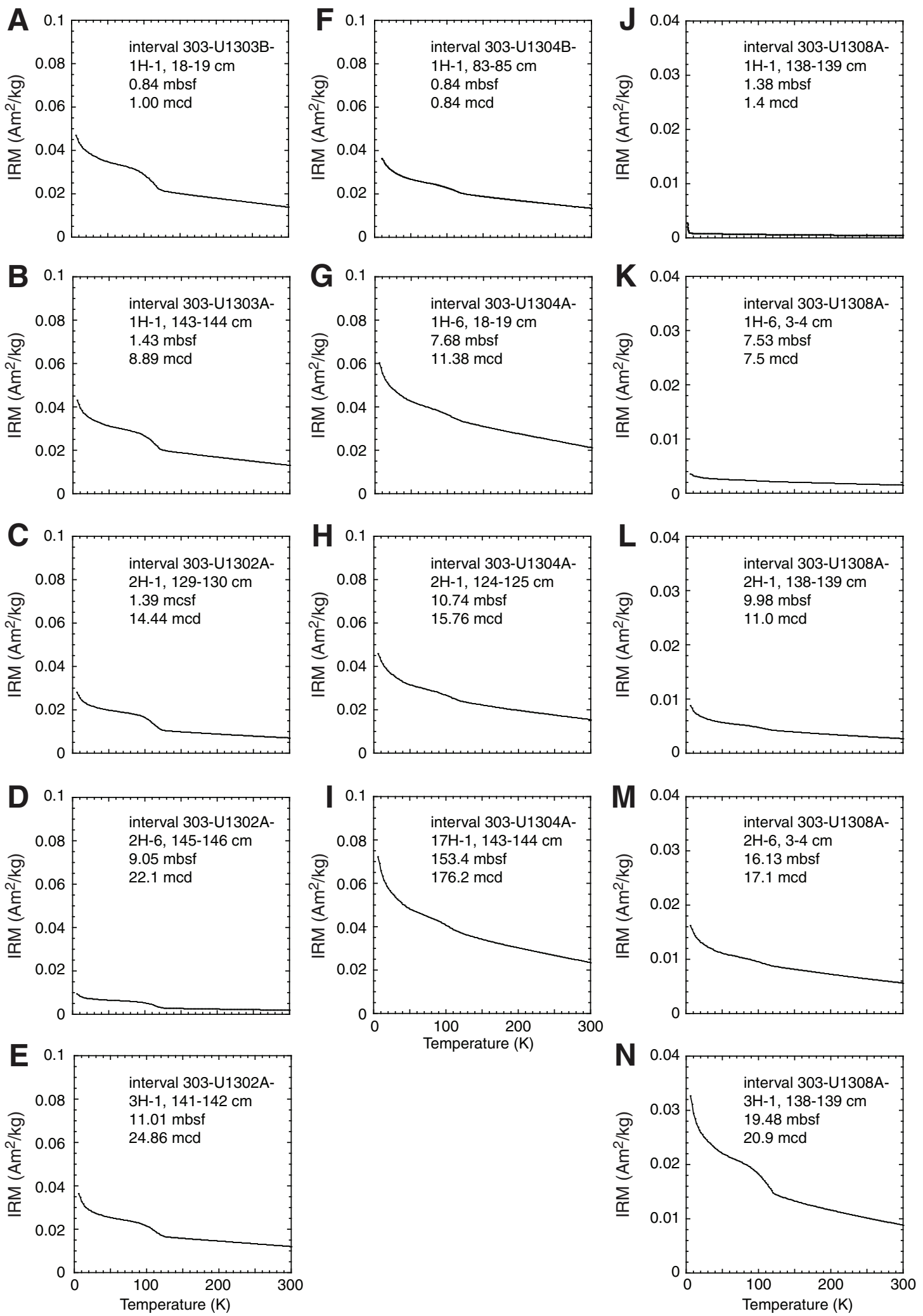
Figure F6. Results of high-temperature magnetometry.

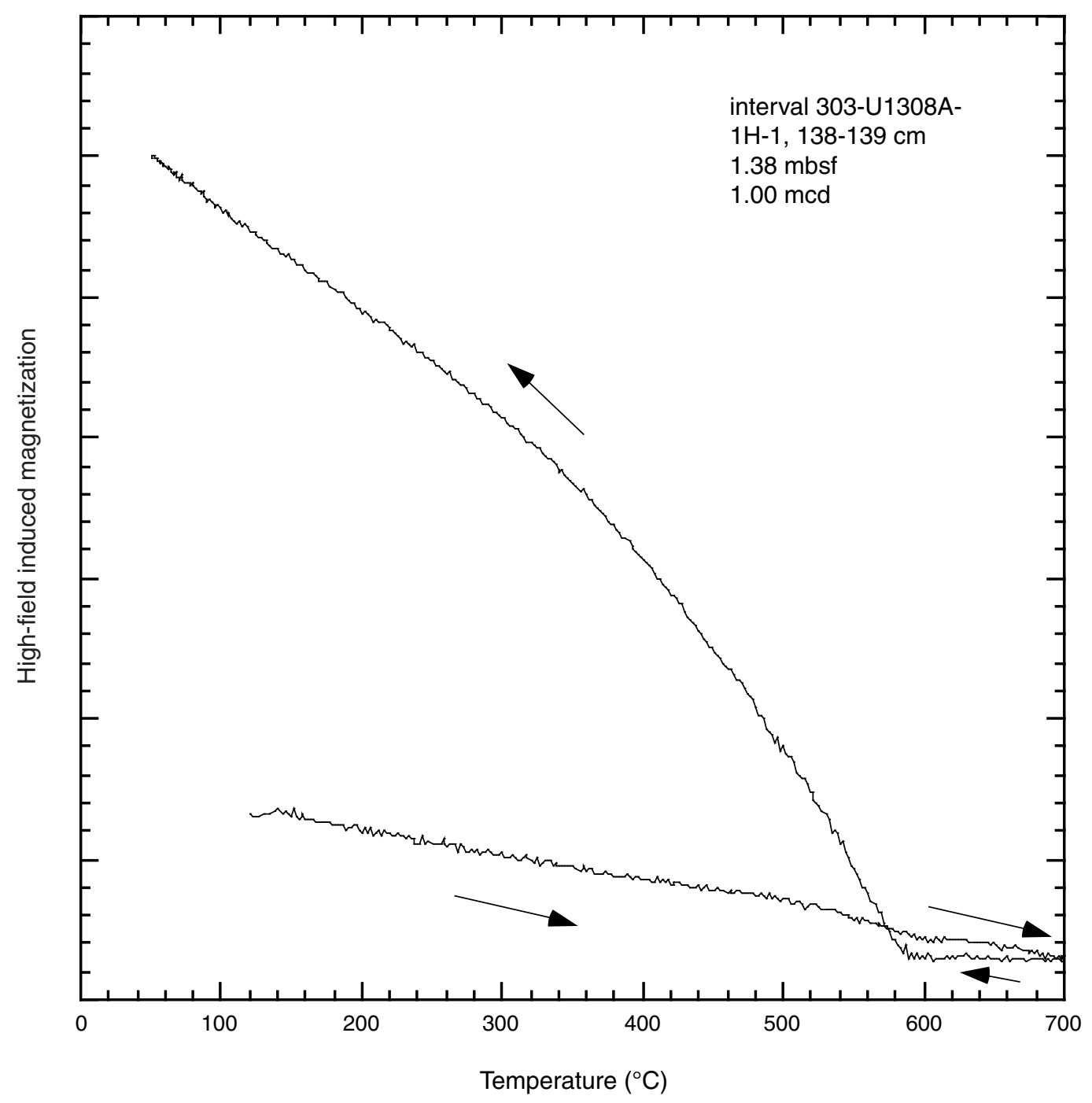


Table T1. Rock magnetic parameters and lithology, Sites U1302-U1304 and U1308. (See table notes.) (Continued on next four pages.)

\begin{tabular}{|c|c|c|c|c|c|c|c|}
\hline \multirow{2}{*}{$\begin{array}{l}\text { Core, section, } \\
\text { interval }(\mathrm{cm})\end{array}$} & \multicolumn{2}{|c|}{ Depth } & \multirow{2}{*}{$\begin{array}{l}\text { Sample } \\
\text { weight } \\
\text { (g) }\end{array}$} & \multirow{2}{*}{$\begin{array}{c}\text { NRM } \\
\left(\mathrm{Am}^{2} / \mathrm{kg}\right)\end{array}$} & \multirow{2}{*}{$\begin{array}{c}\chi \\
\left(m^{3} / \mathrm{kg}\right)\end{array}$} & \multirow{2}{*}{$\begin{array}{c}\chi_{\text {ARM }} \\
\left(\mathrm{m}^{3} / \mathrm{kg}\right)\end{array}$} & \multirow[b]{2}{*}{ Lithology } \\
\hline & (mbsf) & (mcd) & & & & & \\
\hline \multicolumn{8}{|l|}{ 303-U1302A- } \\
\hline $4 \mathrm{H}-3,78-80$ & 22.89 & 38.72 & 8.86 & 1.59.E-04 & $7.96 \mathrm{E}-07$ & $4.52 \mathrm{E}-04$ & Foraminifer silty sand \\
\hline $4 \mathrm{H}-4,74-76$ & 24.35 & 40.18 & 7.40 & 2.43.E-04 & $8.19 \mathrm{E}-07$ & $6.75 \mathrm{E}-04$ & Silty clay \\
\hline $4 \mathrm{H}-5,74-76$ & 25.85 & 41.68 & 8.52 & 5.86.E-05 & $4.16 \mathrm{E}-07$ & $3.18 \mathrm{E}-04$ & Foraminifer sand \\
\hline $6 \mathrm{H}-3,74-77$ & 41.85 & 60.17 & 7.62 & 1.09.E-04 & $4.50 \mathrm{E}-07$ & $3.88 \mathrm{E}-04$ & Nannofossil silty clay \\
\hline $6 \mathrm{H}-4,74-78$ & 43.35 & 61.67 & 8.48 & 1.84.E-04 & $8.23 \mathrm{E}-07$ & $4.61 \mathrm{E}-04$ & Nannofossil silty clay \\
\hline $6 \mathrm{H}-5,74-79$ & 44.85 & 63.17 & 7.04 & 1.48.E-04 & $4.83 \mathrm{E}-07$ & $5.45 \mathrm{E}-04$ & Silty clay nannofossil ooze \\
\hline $6 \mathrm{H}-6,74-78$ & 46.35 & 64.67 & 8.14 & 1.45.E-04 & $6.09 \mathrm{E}-07$ & $6.13 \mathrm{E}-04$ & Silty clay nannofossil ooze \\
\hline $10 \mathrm{H}-2,74-79$ & 78.35 & 102.03 & 9.40 & 1.09.E-04 & 5.47E-07 & $3.24 \mathrm{E}-04$ & Silty clay \\
\hline $10 \mathrm{H}-4,74-79$ & 81.35 & 105.03 & 10.11 & 1.68.E-04 & $7.52 \mathrm{E}-07$ & $4.46 \mathrm{E}-04$ & Silty clay \\
\hline $10 \mathrm{H}-5,74-80$ & 82.85 & 106.53 & 9.52 & 2.22.E-04 & $8.21 \mathrm{E}-07$ & $5.63 \mathrm{E}-04$ & Nannofossil clay \\
\hline $10 \mathrm{H}-6,74-80$ & 84.35 & 108.03 & 10.03 & 6.79.E-05 & $5.32 \mathrm{E}-07$ & $2.83 \mathrm{E}-04$ & Matrix-supported interaclast conglomerate \\
\hline $11 \mathrm{H}-1,74-81$ & 86.35 & 110.03 & 9.34 & 7.01.E-05 & 4.54E-07 & $2.14 \mathrm{E}-04$ & Matrix-supported interaclast conglomerate \\
\hline $11 \mathrm{H}-2,74-81$ & 87.85 & 111.53 & 11.13 & 2.48.E-04 & $1.03 \mathrm{E}-06$ & $3.34 \mathrm{E}-04$ & Matrix-supported interaclast conglomerate \\
\hline $11 \mathrm{H}-4,74-82$ & 90.85 & 114.53 & 10.98 & 1.10.E-04 & $7.20 \mathrm{E}-07$ & $2.91 \mathrm{E}-04$ & Matrix-supported interaclast conglomerate \\
\hline $11 \mathrm{H}-5,74-82$ & 92.35 & 116.03 & 10.62 & 1.01.E-04 & $5.34 \mathrm{E}-07$ & $1.77 \mathrm{E}-04$ & Silty clay \\
\hline $11 \mathrm{H}-6,74-83$ & 93.85 & 117.53 & 10.64 & 5.77.E-05 & $5.17 \mathrm{E}-07$ & $3.16 \mathrm{E}-04$ & Calcareous ooze \\
\hline $12 \mathrm{H}-2,74-83$ & 97.35 & 121.03 & 10.73 & 2.74.E-05 & $3.06 \mathrm{E}-07$ & $1.73 \mathrm{E}-04$ & Matrix-supported interaclast conglomerate \\
\hline $12 \mathrm{H}-3,74-84$ & 98.85 & 122.53 & 10.87 & 9.38.E-05 & $5.10 \mathrm{E}-07$ & $2.73 \mathrm{E}-04$ & Matrix-supported interaclast conglomerate \\
\hline $12 \mathrm{H}-5,74-84$ & 101.85 & 125.53 & 8.88 & 8.56.E-05 & $6.19 \mathrm{E}-07$ & $3.14 \mathrm{E}-04$ & Matrix-supported interaclast conglomerate \\
\hline \multicolumn{8}{|l|}{ 303-U1302B- } \\
\hline $1 \mathrm{H}-3,74-76$ & 3.75 & 12.65 & 7.57 & 2.09.E-04 & $8.72 \mathrm{E}-07$ & $5.68 \mathrm{E}-04$ & Nannofossil ooze silt \\
\hline $1 \mathrm{H}-4,74-76$ & 5.25 & 14.15 & 7.79 & 1.83.E-04 & $8.89 \mathrm{E}-07$ & $4.20 \mathrm{E}-04$ & Nannofossil ooze silt \\
\hline $1 \mathrm{H}-4,74-76$ & 6.75 & 15.65 & 9.03 & 1.43.E-04 & $8.69 \mathrm{E}-07$ & $5.20 \mathrm{E}-04$ & Nannofossil ooze silt \\
\hline $2 \mathrm{H}-4,74-76$ & 14.95 & 24.39 & 11.42 & 8.70.E-05 & $5.33 \mathrm{E}-07$ & $1.65 \mathrm{E}-04$ & Silty clay \\
\hline $2 \mathrm{H}-5,74-76$ & 16.45 & 25.89 & 9.27 & 1.00.E-04 & $4.13 \mathrm{E}-07$ & $3.24 \mathrm{E}-04$ & Silty clay \\
\hline $3 \mathrm{H}-2,74-76$ & 21.45 & 32.00 & 9.11 & 8.40.E-05 & $4.24 \mathrm{E}-07$ & $2.46 \mathrm{E}-04$ & Silty clay nannofossil ooze \\
\hline $3 \mathrm{H}-3,74-76$ & 22.95 & 33.50 & 7.78 & 2.22.E-04 & $8.62 \mathrm{E}-07$ & $4.81 \mathrm{E}-04$ & Nannofossil silty clay \\
\hline $3 \mathrm{H}-4,74-76$ & 24.45 & 35.00 & 7.23 & 1.64.E-04 & $7.85 \mathrm{E}-07$ & $6.71 \mathrm{E}-04$ & Nannofossil silty clay \\
\hline $3 \mathrm{H}-5,74-76$ & 25.95 & 36.50 & 6.68 & 1.97.E-04 & $6.80 \mathrm{E}-07$ & $3.79 \mathrm{E}-04$ & Silty clay \\
\hline $3 \mathrm{H}-6,74-76$ & 27.45 & 38.00 & 6.63 & 2.38.E-04 & $8.73 \mathrm{E}-07$ & $6.86 \mathrm{E}-04$ & Silty clay \\
\hline $4 \mathrm{H}-2,74-76$ & 30.95 & 42.72 & 10.26 & 1.05.E-04 & $7.54 \mathrm{E}-07$ & $1.61 \mathrm{E}-04$ & Nannofossil silty clay \\
\hline $4 \mathrm{H}-3,74-76$ & 32.45 & 44.22 & 10.34 & 1.08.E-04 & $6.13 \mathrm{E}-07$ & $2.96 \mathrm{E}-04$ & Silty clay \\
\hline $4 \mathrm{H}-4,74-76$ & 33.95 & 45.72 & 7.42 & 1.51.E-04 & $8.14 \mathrm{E}-07$ & $6.77 \mathrm{E}-04$ & Nannofossil ooze silt \\
\hline $5 \mathrm{H}-4,74-76$ & 43.45 & 57.93 & 8.53 & 1.31.E-04 & $4.82 \mathrm{E}-07$ & $3.85 \mathrm{E}-04$ & Nannofossil ooze with silty clay \\
\hline $5 \mathrm{H}-5,70-72$ & 44.95 & 59.43 & 10.40 & 1.21.E-04 & 7.36E-07 & $2.76 \mathrm{E}-04$ & Nannofossil ooze with silty clay \\
\hline $6 \mathrm{H}-3,74-76$ & 51.45 & 65.59 & 12.17 & 7.84.E-05 & $4.76 \mathrm{E}-07$ & $8.25 \mathrm{E}-05$ & Silty clay \\
\hline $6 \mathrm{H}-4,74-76$ & 52.95 & 67.09 & 9.63 & 1.90.E-04 & $8.51 \mathrm{E}-07$ & $5.35 \mathrm{E}-04$ & Silty clay \\
\hline $7 \mathrm{H}-3,74-76$ & 60.95 & 75.68 & 11.29 & 8.52.E-05 & $7.88 \mathrm{E}-07$ & $1.43 \mathrm{E}-04$ & Sandy clay calcareous ooze \\
\hline $7 \mathrm{H}-5,74-76$ & 63.95 & 78.68 & 9.35 & 1.25.E-04 & $8.61 \mathrm{E}-07$ & $3.14 \mathrm{E}-04$ & Clay \\
\hline $8 \mathrm{H}-2,74-76$ & 68.95 & 85.74 & 7.55 & 2.57.E-04 & $8.83 \mathrm{E}-07$ & $6.69 \mathrm{E}-04$ & Silty clay \\
\hline $8 \mathrm{H}-3,74-76$ & 70.45 & 87.24 & 9.47 & 1.11.E-04 & 5.97E-07 & $4.09 \mathrm{E}-04$ & Silty clay \\
\hline $8 \mathrm{H}-4,74-76$ & 71.95 & 88.74 & 9.30 & 1.80.E-04 & $8.74 \mathrm{E}-07$ & $6.33 \mathrm{E}-04$ & Silty clay \\
\hline $8 \mathrm{H}-5,74-76$ & 73.45 & 90.24 & 9.69 & 1.35.E-04 & $6.41 \mathrm{E}-07$ & $6.31 \mathrm{E}-04$ & Clay with foraminifers \\
\hline $9 \mathrm{H}-3,74-76$ & 79.95 & 98.03 & 10.57 & 1.54.E-04 & $7.83 \mathrm{E}-07$ & $4.88 \mathrm{E}-04$ & Clay \\
\hline $9 \mathrm{H}-4,74-76$ & 81.45 & 99.53 & 9.50 & 1.32.E-04 & $5.31 \mathrm{E}-07$ & $4.25 \mathrm{E}-04$ & Silty clay \\
\hline $9 \mathrm{H}-5,74-76$ & 82.95 & 101.03 & 12.89 & 9.04.E-05 & $5.06 \mathrm{E}-07$ & $9.23 \mathrm{E}-05$ & Silty clay \\
\hline \multicolumn{8}{|l|}{ 303-U1302C- } \\
\hline $1 \mathrm{H}-4,74-76$ & 5.25 & 9.27 & 7.65 & 1.40.E-04 & $6.44 \mathrm{E}-07$ & $4.94 \mathrm{E}-04$ & Silty clay \\
\hline $1 \mathrm{H}-5,74-76$ & 6.75 & 10.77 & 8.62 & 1.16.E-04 & $5.88 \mathrm{E}-07$ & $4.28 \mathrm{E}-04$ & Silty clay \\
\hline $1 \mathrm{H}-6,74-76$ & 8.25 & 12.27 & 7.72 & 1.74.E-04 & 7.49E-07 & $5.23 \mathrm{E}-04$ & Silty clay \\
\hline $2 \mathrm{H}-4,74-76$ & 14.75 & 18.81 & 7.35 & 1.95.E-04 & $9.14 \mathrm{E}-07$ & $6.32 \mathrm{E}-04$ & Silty clay \\
\hline $2 \mathrm{H}-6,74-76$ & 17.75 & 21.81 & 8.16 & 1.05.E-04 & $4.89 \mathrm{E}-07$ & $4.24 \mathrm{E}-04$ & Silty clay foraminifers \\
\hline $3 \mathrm{H}-2,74-76$ & 21.25 & 25.49 & 8.23 & 1.85.E-04 & $7.56 \mathrm{E}-07$ & $6.43 \mathrm{E}-04$ & Silty clay \\
\hline $3 \mathrm{H}-2,74-76$ & 22.75 & 26.99 & 8.49 & 1.55.E-04 & $4.86 \mathrm{E}-07$ & $1.86 \mathrm{E}-04$ & Silty clay \\
\hline $7 \mathrm{H}-3,74-76$ & 60.75 & 71.04 & 10.33 & 1.29.E-04 & $2.59 \mathrm{E}-06$ & $2.25 \mathrm{E}-04$ & Silty clay nannofossil ooze \\
\hline $7 \mathrm{H}-4,74-76$ & 62.25 & 72.54 & 8.33 & 1.83.E-04 & $8.39 \mathrm{E}-07$ & $5.01 \mathrm{E}-04$ & Silty clay nannofossil ooze \\
\hline $7 \mathrm{H}-5,74-76$ & 63.75 & 74.04 & 7.37 & 1.33.E-04 & 4.99E-07 & $4.30 \mathrm{E}-04$ & Silty clay nannofossil ooze \\
\hline $8 \mathrm{H}-2,74-76$ & 68.75 & 80.10 & 8.84 & 1.39.E-04 & $7.73 \mathrm{E}-07$ & $5.14 \mathrm{E}-04$ & Silty clay nannofossil ooze \\
\hline $8 \mathrm{H}-3,74-76$ & 70.25 & 81.60 & 8.10 & 1.78.E-04 & $6.98 \mathrm{E}-07$ & $5.81 \mathrm{E}-04$ & Silty clay nannofossil ooze \\
\hline $8 \mathrm{H}-4,74-76$ & 71.75 & 83.10 & 9.01 & 1.40.E-04 & $6.73 \mathrm{E}-07$ & $3.97 \mathrm{E}-04$ & Silty clay nannofossil ooze \\
\hline $8 \mathrm{H}-5,74-75$ & 73.25 & 84.60 & 10.42 & 1.43.E-04 & $6.54 \mathrm{E}-07$ & $3.77 \mathrm{E}-04$ & Silty clay nannofossil ooze \\
\hline $9 \mathrm{H}-2,74-76$ & 78.25 & 91.14 & 10.99 & 1.14.E-04 & $6.28 \mathrm{E}-07$ & $2.52 \mathrm{E}-04$ & Silty clay nannofossil ooze \\
\hline $9 \mathrm{H}-3,74-76$ & 79.75 & 92.64 & 10.33 & 9.73.E-05 & $5.41 \mathrm{E}-07$ & $3.02 \mathrm{E}-04$ & Silty clay nannofossil ooze \\
\hline $9 \mathrm{H}-4,74-76$ & 81.25 & 94.14 & 11.44 & 1.00.E-04 & $5.59 \mathrm{E}-07$ & $1.77 \mathrm{E}-04$ & Clay \\
\hline
\end{tabular}


Table T1 (continued). (Continued on next page.)

\begin{tabular}{|c|c|c|c|c|c|c|c|}
\hline \multirow{2}{*}{$\begin{array}{l}\text { Core, section, } \\
\text { interval }(\mathrm{cm})\end{array}$} & \multicolumn{2}{|c|}{ Depth } & \multirow{2}{*}{$\begin{array}{l}\text { Sample } \\
\text { weight } \\
\text { (g) }\end{array}$} & \multirow{2}{*}{$\begin{array}{c}\mathrm{NRM} \\
\left(\mathrm{Am}^{2} / \mathrm{kg}\right)\end{array}$} & \multirow{2}{*}{$\begin{array}{c}\chi \\
\left(\mathrm{m}^{3} / \mathrm{kg}\right)\end{array}$} & \multirow{2}{*}{$\begin{array}{c}\chi_{\text {ARM }} \\
\left(\mathrm{m}^{3} / \mathrm{kg}\right)\end{array}$} & \multirow[b]{2}{*}{ Lithology } \\
\hline & (mbsf) & $(\mathrm{mcd})$ & & & & & \\
\hline \multicolumn{8}{|l|}{ 303-U1302D- } \\
\hline $1 \mathrm{H}-1,84-86$ & 0.85 & 0.85 & 6.12 & 1.71.E-04 & $6.05 \mathrm{E}-07$ & $6.52 \mathrm{E}-04$ & Nannofossil ooze with clay \\
\hline $1 \mathrm{H}-2,74-76$ & 2.35 & 2.35 & 7.34 & 2.30.E-04 & $8.99 \mathrm{E}-07$ & $6.50 \mathrm{E}-04$ & Silty clay \\
\hline \multicolumn{8}{|l|}{ 303-1303A- } \\
\hline $5 \mathrm{H}-2,74-76$ & 39.15 & 50.28 & 8.38 & 1.73.E-04 & 8.66E-07 & $6.13 \mathrm{E}-04$ & Silty nannofossil ooze \\
\hline $5 \mathrm{H}-3,74-76$ & 40.65 & 51.78 & 10.84 & 7.87.E-05 & $4.00 \mathrm{E}-07$ & $1.73 \mathrm{E}-04$ & Silty nannofossil ooze \\
\hline $5 \mathrm{H}-4,74-76$ & 42.15 & 53.28 & 7.33 & 2.00.E-04 & $6.01 \mathrm{E}-07$ & $5.47 \mathrm{E}-04$ & Silty nannofossil ooze \\
\hline \multicolumn{8}{|l|}{ 303-1303B- } \\
\hline 1H-5, 74-76 & 6.75 & 7.57 & 8.86 & 2.10.E-04 & $9.56 \mathrm{E}-07$ & 4.54E-04 & Silty nannofossil ooze \\
\hline $1 \mathrm{H}-6,74-76$ & 8.25 & 9.07 & 7.05 & 1.49.E-04 & $6.70 \mathrm{E}-07$ & $5.23 \mathrm{E}-04$ & Silty nannofossil ooze \\
\hline \multicolumn{8}{|l|}{ 303-1304A- } \\
\hline $1 \mathrm{H}-1,83-85$ & 0.84 & 4.54 & 5.06 & 1.77.E-04 & 5.95.E-07 & 6.47.E-04 & Nannofossil ooze \\
\hline $1 \mathrm{H}-3,83-85$ & 3.84 & 7.54 & 5.55 & 2.19.E-04 & 5.71.E-07 & 6.81.E-04 & Nannofossil ooze \\
\hline $1 \mathrm{H}-4,83-85$ & 5.34 & 9.04 & 5.70 & 2.42.E-04 & 5.62.E-07 & 6.11.E-04 & Diatom nannofossil ooze \\
\hline $2 \mathrm{H}-2,83-85$ & 11.84 & 16.86 & 6.12 & 9.11.E-05 & 2.67.E-07 & 3.06.E-04 & Diatom nannofossil ooze \\
\hline $2 \mathrm{H}-3,83-85$ & 13.34 & 18.36 & 6.43 & 5.76.E-05 & 1.67.E-07 & 2.26.E-04 & Diatom nannofossil ooze \\
\hline $2 \mathrm{H}-4,83-85$ & 14.84 & 19.86 & 4.25 & 1.67.E-05 & 9.64.E-08 & 1.37.E-04 & Diatom ooze \\
\hline $2 \mathrm{H}-5,83-85$ & 16.34 & 21.36 & 5.61 & 1.83.E-04 & 7.25.E-07 & 8.46.E-04 & Diatom nannofossil ooze \\
\hline $2 \mathrm{H}-6,83-85$ & 17.84 & 22.86 & 6.03 & 1.77.E-04 & 6.12.E-07 & 7.28.E-04 & Diatom nannofossil ooze \\
\hline $3 \mathrm{H}-2,83-85$ & 21.34 & 28.03 & 4.93 & 4.30.E-04 & 1.41.E-06 & 1.34.E-03 & Nannofossil ooze \\
\hline $3 \mathrm{H}-3,83-85$ & 22.84 & 29.53 & 6.20 & 1.99.E-04 & 5.28.E-07 & 5.82.E-04 & Nannofossil ooze \\
\hline $3 \mathrm{H}-4,83-85$ & 24.34 & 31.03 & 7.83 & 1.07.E-04 & 5.10.E-07 & 5.23.E-04 & Nannofossil ooze \\
\hline $3 \mathrm{H}-5,83-85$ & 25.84 & 32.53 & 8.02 & 2.55.E-04 & 5.67.E-07 & 5.62.E-04 & Nannofossil ooze \\
\hline $3 \mathrm{H}-6,83-85$ & 27.34 & 34.03 & 6.73 & 7.64.E-05 & 3.60.E-07 & 3.54.E-04 & Nannofossil ooze \\
\hline $4 \mathrm{H}-3,83-85$ & 32.34 & 39.54 & 7.13 & 1.12.E-04 & 5.21.E-07 & 5.86.E-04 & Nannofossil ooze \\
\hline $4 \mathrm{H}-4,83-85$ & 33.84 & 41.04 & 7.17 & 3.00.E-04 & 7.51.E-07 & 8.50.E-04 & Nannofossil ooze \\
\hline $4 \mathrm{H}-5,83-85$ & 35.34 & 42.54 & 7.98 & 1.52.E-04 & 5.82.E-07 & 5.95.E-04 & Nannofossil ooze \\
\hline $5 \mathrm{H}-4,83-85$ & 43.34 & 51.60 & 5.63 & 4.03.E-05 & 2.10.E-07 & 1.75.E-04 & Diatom ooze \\
\hline $5 \mathrm{H}-5,83-85$ & 44.84 & 53.10 & 7.41 & 1.38.E-04 & 5.58.E-07 & 5.37.E-04 & Nannofossil ooze \\
\hline $6 \mathrm{H}-3,83-85$ & 51.34 & 60.54 & 5.59 & 2.48.E-04 & 6.19.E-07 & 6.38.E-04 & Diatom nannofossil ooze \\
\hline $6 \mathrm{H}-4,83-85$ & 52.84 & 62.04 & 6.15 & 6.68.E-05 & 2.04.E-07 & 3.07.E-04 & Diatom nannofossil ooze \\
\hline $6 \mathrm{H}-5,83-85$ & 54.34 & 63.54 & 7.13 & 1.47.E-05 & 6.00.E-08 & 8.38.E-05 & Diatom nannofossil ooze \\
\hline $7 \mathrm{H}-1,83-85$ & 57.84 & 69.60 & 8.89 & 9.71.E-05 & 3.37.E-07 & 3.72.E-04 & Nannofossil ooze \\
\hline $7 \mathrm{H}-2,83-85$ & 57.84 & 69.60 & 8.79 & 8.27.E-05 & 3.02.E-07 & 3.79.E-04 & Nannofossil ooze \\
\hline $7 \mathrm{H}-3,83-85$ & 60.84 & 72.60 & 8.19 & 1.55.E-04 & 7.16.E-07 & 6.71.E-04 & Silty clay nannofossil ooze \\
\hline $7 \mathrm{H}-5,83-85$ & 62.34 & 74.10 & 8.07 & 1.42.E-04 & 6.39.E-07 & 5.66.E-04 & Silty clay nannofossil ooze \\
\hline $8 \mathrm{H}-2,83-85$ & 68.84 & 81.94 & 7.35 & 1.88.E-04 & 6.04.E-07 & 6.41.E-04 & Nannofossil ooze \\
\hline $8 \mathrm{H}-3,83-85$ & 70.34 & 83.44 & 7.29 & 1.11.E-04 & 4.79.E-07 & 5.46.E-04 & Diatom nannofossil ooze \\
\hline $8 \mathrm{H}-4,83-85$ & 71.84 & 84.94 & 5.08 & 1.41.E-04 & 3.40.E-07 & 3.65.E-04 & Diatom nannofossil ooze \\
\hline $8 \mathrm{H}-5,83-85$ & 73.34 & 86.44 & 6.23 & 1.53.E-04 & 6.07.E-07 & 5.95.E-04 & Nannofossil ooze \\
\hline $9 \mathrm{H}-3,83-85$ & 79.84 & 93.99 & 8.91 & 1.21.E-05 & 1.00.E-07 & 5.02.E-05 & Diatom nannofossil ooze \\
\hline $9 \mathrm{H}-4,83-85$ & 81.34 & 95.49 & 8.02 & 8.66.E-05 & 4.14.E-07 & 3.46.E-04 & Nannofossil diatom ooze \\
\hline $10 \mathrm{H}-3,83-85$ & 89.36 & 104.02 & 7.03 & 1.20.E-05 & 1.07.E-07 & 7.57.E-05 & Nannofossil diatom ooze \\
\hline $10 \mathrm{H}-4,83-85$ & 90.88 & 105.54 & 10.31 & 4.67.E-05 & 1.58.E-07 & 1.28.E-04 & Nannofossil diatom ooze \\
\hline $11 \mathrm{H}-2,83-85$ & 97.34 & 113.26 & 8.25 & 2.17.E-04 & 9.23.E-07 & 7.94.E-04 & Clay \\
\hline $11 \mathrm{H}-3,83-85$ & 98.84 & 114.76 & 5.64 & 1.17.E-05 & 4.02.E-08 & 2.23.E-05 & Diatom ooze \\
\hline $11 \mathrm{H}-4,83-85$ & 100.34 & 116.26 & 8.15 & 2.78.E-04 & 8.56.E-07 & 8.09.E-04 & Diatom nannofossil ooze \\
\hline $11 \mathrm{H}-5,83-85$ & 101.84 & 117.76 & 6.44 & 2.95.E-04 & 8.45.E-07 & 8.82.E-04 & Nannofossil ooze \\
\hline $11 \mathrm{H}-6,83-85$ & 103.34 & 119.26 & 6.06 & 3.27.E-04 & 8.75.E-07 & 8.90.E-04 & Clay \\
\hline $12 \mathrm{H}-3,83-85$ & 108.34 & 125.09 & 8.81 & 4.98.E-05 & 2.76.E-07 & 2.47.E-04 & Nannofossil ooze \\
\hline $12 \mathrm{H}-4,83-85$ & 109.84 & 126.59 & 7.43 & 2.49.E-04 & 9.51.E-07 & 9.41.E-04 & Clay \\
\hline $12 \mathrm{H}-5,83-85$ & 111.34 & 128.09 & 7.81 & 1.77.E-04 & 8.82.E-07 & 9.37.E-04 & Clay \\
\hline $13 \mathrm{H}-3,83-85$ & 117.84 & 135.96 & 7.82 & 2.84.E-04 & 9.50.E-07 & 1.05.E-03 & Nannofossil ooze \\
\hline $13 \mathrm{H}-4,83-85$ & 119.34 & 137.46 & 8.29 & 3.76.E-04 & 1.04.E-06 & 1.02.E-03 & Nannofossil ooze \\
\hline $13 \mathrm{H}-5,83-85$ & 120.84 & 138.96 & 6.15 & 6.71.E-05 & 8.41.E-07 & 9.78.E-04 & Nannofossil ooze \\
\hline $13 \mathrm{H}-6,83-85$ & 122.34 & 140.46 & 8.89 & 2.36.E-05 & 4.27.E-07 & 5.19.E-04 & Clay \\
\hline $14 \mathrm{H}-2,83-85$ & 125.84 & 145.44 & 7.80 & 7.80.E-05 & 3.09.E-07 & 4.57.E-04 & Nannofossil ooze \\
\hline $14 \mathrm{H}-3,83-85$ & 127.34 & 146.94 & 7.24 & 1.75.E-04 & 5.62.E-07 & 7.01.E-04 & Nannofossil ooze \\
\hline $14 \mathrm{H}-4,83-85$ & 128.84 & 148.44 & 6.72 & 2.12.E-05 & 4.57.E-07 & 4.28.E-04 & Diatom ooze \\
\hline $14 \mathrm{H}-5,83-85$ & 130.34 & 149.94 & 7.60 & 1.54.E-04 & 8.13.E-07 & 9.36.E-04 & Silty clay nannofossil ooze \\
\hline $15 \mathrm{H}-3,83-85$ & 136.84 & 156.80 & 8.54 & 4.31.E-05 & 8.51.E-07 & 7.97.E-04 & Silty clay nannofossil ooze \\
\hline 303-1304B- & & & & & & & \\
\hline $1 \mathrm{H}-1,83-85$ & 0.84 & 0.84 & 6.29 & 1.57.E-05 & 2.29.E-07 & 2.57.E-04 & Nannofossil ooze \\
\hline $1 \mathrm{H}-3,83-85$ & 3.84 & 3.84 & 4.75 & 5.02.E-05 & 1.25.E-07 & 2.11.E-04 & Silty clay \\
\hline $2 \mathrm{H}-2,83-85$ & 10.54 & 10.36 & 6.94 & 1.63.E-04 & 5.39.E-07 & 6.10.E-04 & Nannofossil ooze \\
\hline $2 \mathrm{H}-3,83-85$ & 12.04 & 11.86 & 7.09 & 2.32.E-04 & 6.39.E-07 & 6.34.E-04 & Silty clay \\
\hline $2 \mathrm{H}-4,83-85$ & 13.54 & 13.36 & 6.89 & 1.86.E-04 & 7.29.E-07 & 7.74.E-04 & Nannofossil ooze \\
\hline
\end{tabular}


Table T1 (continued). (Continued on next page.)

\begin{tabular}{|c|c|c|c|c|c|c|c|}
\hline \multirow{2}{*}{$\begin{array}{l}\text { Core, section, } \\
\text { interval }(\mathrm{cm})\end{array}$} & \multicolumn{2}{|c|}{ Depth } & \multirow{2}{*}{$\begin{array}{l}\text { Sample } \\
\text { weight } \\
\text { (g) }\end{array}$} & \multirow{2}{*}{$\begin{array}{c}\mathrm{NRM} \\
\left(\mathrm{Am}^{2} / \mathrm{kg}\right)\end{array}$} & \multirow{2}{*}{$\begin{array}{c}\chi \\
\left(\mathrm{m}^{3} / \mathrm{kg}\right)\end{array}$} & \multirow{2}{*}{$\begin{array}{c}\chi_{\text {ARM }} \\
\left(\mathrm{m}^{3} / \mathrm{kg}\right)\end{array}$} & \multirow[b]{2}{*}{ Lithology } \\
\hline & (mbsf) & $(\mathrm{mcd})$ & & & & & \\
\hline $2 \mathrm{H}-5,83-85$ & 15.04 & 14.86 & 6.30 & 7.77.E-05 & 2.86.E-07 & 4.03.E-04 & Nannofossil ooze \\
\hline $3 \mathrm{H}-4,83-85$ & 23.04 & 23.91 & 6.86 & 2.16.E-04 & 6.65.E-07 & 6.85.E-04 & Nannofossil ooze \\
\hline $3 \mathrm{H}-5,83-85$ & 24.54 & 25.41 & 5.87 & 1.80.E-04 & 1.22.E-06 & 1.21.E-03 & Diatom nannofossil ooze \\
\hline $3 \mathrm{H}-6,83-85$ & 26.04 & 26.91 & 6.52 & 2.35.E-04 & 6.33.E-07 & 7.04.E-04 & Nannofossil ooze \\
\hline $4 \mathrm{H}-4,83-85$ & 32.53 & 34.83 & 6.74 & 1.93.E-04 & 5.13.E-07 & 5.41.E-04 & Diatom silty clay \\
\hline $4 \mathrm{H}-5,83-85$ & 34.03 & 36.33 & 7.03 & 2.28.E-04 & 8.65.E-07 & 8.70.E-04 & Diatom nannofossil ooze \\
\hline $4 \mathrm{H}-6,83-85$ & 35.53 & 37.83 & 8.00 & 1.87.E-04 & 5.61.E-07 & 6.68.E-04 & Nannofossil ooze \\
\hline $5 \mathrm{H}-2,83-85$ & 39.04 & 43.47 & 7.13 & 2.68.E-04 & 7.80.E-07 & 7.85.E-04 & Nannofossil diatom ooze \\
\hline $5 \mathrm{H}-4,83-85$ & 42.04 & 46.47 & 6.19 & 4.99.E-06 & 1.47.E-08 & 8.98.E-06 & Clay diatom ooze \\
\hline $5 \mathrm{H}-5,83-85$ & 43.54 & 47.97 & 5.53 & 5.18.E-05 & 2.90.E-07 & 2.92.E-04 & Diatom silty clay \\
\hline $5 \mathrm{H}-6,83-85$ & 45.04 & 49.47 & 6.77 & 1.70.E-04 & 5.98.E-07 & 6.25.E-04 & Diatom nannofossil ooze \\
\hline $6 \mathrm{H}-3,83-85$ & 50.04 & 54.93 & 4.89 & 5.84.E-05 & 1.47.E-07 & 2.43.E-04 & Diatom nannofossil ooze \\
\hline $6 \mathrm{H}-4,83-85$ & 51.54 & 56.43 & 4.94 & 9.80.E-07 & 3.36.E-09 & 3.48.E-06 & Nannofossil diatom ooze \\
\hline $6 \mathrm{H}-5,83-85$ & 53.04 & 57.93 & 6.48 & 2.33.E-06 & 1.12.E-08 & 5.97.E-06 & Nannofossil diatom ooze \\
\hline $6 \mathrm{H}-6,83-85$ & 54.54 & 59.43 & 5.90 & 1.05.E-04 & 3.63.E-07 & 4.37.E-04 & Nannofossil ooze \\
\hline $7 \mathrm{H}-3,83-85$ & 59.54 & 64.64 & 7.52 & 2.03.E-05 & 8.12.E-08 & 1.66.E-04 & Nannofossil diatom ooze \\
\hline $7 \mathrm{H}-4,83-85$ & 61.03 & 66.13 & 8.42 & 3.13.E-05 & 1.14.E-07 & 2.09.E-04 & Nannofossil diatom ooze \\
\hline $7 \mathrm{H}-5,83-85$ & 62.52 & 67.62 & 7.83 & 4.57.E-05 & 1.77.E-07 & 2.42.E-04 & Nannofossil diatom ooze \\
\hline $7 \mathrm{H}-6,83-85$ & 64.01 & 69.11 & 7.42 & 7.91.E-05 & 3.04.E-07 & 3.47.E-04 & Nannofossil ooze \\
\hline $8 \mathrm{H}-2,83-85$ & 67.54 & 75.96 & 7.72 & 7.57.E-05 & 3.28.E-07 & 3.78.E-04 & Diatom nannofossil ooze \\
\hline $8 \mathrm{H}-3,83-85$ & 69.04 & 77.46 & 6.11 & 3.88.E-04 & 6.43.E-07 & 6.41.E-04 & Nannofossil ooze \\
\hline $8 \mathrm{H}-4,83-85$ & 70.54 & 78.96 & 6.35 & 1.18.E-04 & 6.65.E-07 & 6.74.E-04 & Nannofossil ooze \\
\hline $8 \mathrm{H}-5,83-85$ & 72.04 & 80.46 & 7.49 & 1.24.E-04 & 5.04.E-07 & 5.22.E-04 & Nannofossil ooze \\
\hline $8 \mathrm{H}-6,83-85$ & 73.54 & 81.96 & 6.89 & 1.50.E-04 & 5.69.E-07 & 6.02.E-04 & Nannofossil ooze \\
\hline $9 \mathrm{H}-3,83-85$ & 78.53 & 88.15 & 7.45 & 3.95.E-05 & 2.24.E-07 & 2.75.E-04 & Nannofossil ooze \\
\hline $9 \mathrm{H}-4,83-85$ & 80.02 & 89.64 & 7.50 & 4.97.E-06 & 3.85.E-08 & 3.13.E-05 & Diatom nannofossil ooze \\
\hline $9 \mathrm{H}-5,83-85$ & 81.51 & 91.13 & 6.88 & 2.49.E-05 & 1.41.E-07 & 2.08.E-04 & Nannofossil ooze \\
\hline $10 \mathrm{H}-1,83-85$ & 85.04 & 95.84 & 8.25 & 4.51.E-05 & 2.30.E-07 & 8.76.E-05 & Nannofossil ooze \\
\hline $10 \mathrm{H}-2,83-85$ & 86.54 & 97.34 & 4.03 & 8.72.E-07 & 3.80.E-09 & 5.49.E-06 & Nannofossil diatom ooze \\
\hline $10 \mathrm{H}-3,83-85$ & 88.04 & 98.84 & 4.14 & 1.24.E-06 & 2.60.E-10 & 4.77.E-06 & Nannofossil diatom ooze \\
\hline $10 \mathrm{H}-4,83-85$ & 89.55 & 100.35 & 4.36 & 7.43.E-07 & 3.96.E-09 & 3.68.E-06 & Nannofossil diatom ooze \\
\hline $10 \mathrm{H}-5,83-85$ & 91.05 & 101.85 & 5.03 & 1.52.E-06 & 1.30.E-08 & 5.14.E-06 & Nannofossil diatom ooze \\
\hline $10 \mathrm{H}-6,83-85$ & 92.54 & 103.34 & 5.56 & 6.47.E-06 & 4.93.E-08 & 1.45.E-05 & Nannofossil diatom ooze \\
\hline $11 \mathrm{H}-2,83-85$ & 96.04 & 108.03 & 4.23 & 9.21.E-05 & 3.05.E-07 & 3.87.E-04 & Diatom nannofossil ooze \\
\hline $11 \mathrm{H}-3,83-85$ & 97.54 & 109.53 & 5.68 & 1.32.E-05 & 5.02.E-08 & 1.98.E-05 & Nannofossil diatom ooze \\
\hline $11 \mathrm{H}-4,83-85$ & 99.04 & 111.03 & 4.37 & 1.52.E-04 & 4.24.E-07 & 4.89.E-04 & Nannofossil and diatom ooze \\
\hline $11 \mathrm{H}-5,83-85$ & 100.55 & 112.54 & 4.93 & 9.27.E-05 & 3.00.E-07 & 1.86.E-04 & Diatom nannofossil ooze \\
\hline $12 \mathrm{H}-3,83-85$ & 107.04 & 120.25 & 6.19 & 3.15.E-04 & 8.77.E-07 & 9.69.E-04 & Diatom nannofossil ooze \\
\hline $12 \mathrm{H}-4,83-85$ & 108.53 & 121.74 & 7.00 & 1.69.E-04 & 8.33.E-07 & 9.31.E-04 & Diatom nannofossil ooze \\
\hline $12 \mathrm{H}-5,83-85$ & 110.03 & 123.24 & 7.41 & 1.46.E-04 & 5.02.E-07 & 5.18.E-04 & Diatom nannofossil ooze \\
\hline $12 \mathrm{H}-6,83-85$ & 111.53 & 124.74 & 8.41 & 7.50.E-05 & 4.99.E-07 & 3.69.E-04 & Diatom nannofossil ooze \\
\hline $13 \mathrm{H}-2,83-85$ & 115.04 & 129.42 & 5.82 & 2.62.E-04 & 8.60.E-07 & 9.97.E-04 & Nannofossil ooze \\
\hline $13 \mathrm{H}-3,83-85$ & 116.53 & 130.91 & 7.49 & 7.41.E-05 & 3.90.E-07 & 3.25.E-04 & Nannofossil ooze \\
\hline $13 \mathrm{H}-4,83-85$ & 118.02 & 132.40 & 6.59 & 3.48.E-04 & 1.07.E-06 & 1.02.E-03 & Nannofossil silty clay \\
\hline $13 \mathrm{H}-5,83-85$ & 119.51 & 133.89 & 6.33 & 3.26.E-04 & 1.18.E-06 & 1.24.E-03 & Nannofossil ooze \\
\hline $14 \mathrm{H}-3,83-85$ & 126.03 & 141.11 & 7.36 & 5.53.E-05 & 6.64.E-07 & 5.88.E-04 & Diatom nannofossil ooze \\
\hline $14 \mathrm{H}-4,83-85$ & 127.52 & 142.60 & 6.15 & 1.31.E-04 & 8.31.E-07 & 9.86.E-04 & Nannofossil ooze \\
\hline $14 \mathrm{H}-5,83-85$ & 129.02 & 144.10 & 4.86 & 2.79.E-05 & 10.00.E-08 & 7.27.E-05 & Nannofossil ooze \\
\hline $15 \mathrm{H}-2,83-85$ & 134.04 & 150.14 & 5.98 & 1.46.E-04 & 1.31.E-06 & 1.48.E-03 & Nannofossil ooze \\
\hline $15 \mathrm{H}-3,83-85$ & 135.53 & 151.63 & 5.95 & 3.37.E-04 & 1.17.E-06 & 1.45.E-03 & Nannofossil ooze \\
\hline $15 \mathrm{H}-4,83-85$ & 137.02 & 153.12 & 6.26 & 3.35.E-04 & 1.01.E-06 & 1.24.E-03 & Nannofossil ooze \\
\hline $15 \mathrm{H}-5,83-85$ & 138.52 & 154.62 & 7.93 & 5.65.E-05 & 9.28.E-07 & 8.95.E-04 & Diatom nannofossil ooze \\
\hline $15 \mathrm{H}-6,83-85$ & 140.02 & 156.12 & 7.38 & 8.55.E-05 & 9.39.E-07 & 1.01.E-03 & Nannofossil ooze \\
\hline $16 \mathrm{H}-1,83-85$ & 142.04 & 158.95 & 4.91 & 1.38.E-04 & 4.04.E-06 & 5.97.E-04 & Diatom nannofossil ooze \\
\hline $16 \mathrm{H}-2,83-85$ & 143.54 & 160.45 & 6.05 & 4.08.E-05 & 3.87.E-07 & 4.07.E-04 & Nannofossil diatom ooze \\
\hline $16 \mathrm{H}-3,83-85$ & 145.05 & 161.96 & 6.71 & 1.39.E-04 & 6.14.E-07 & 7.68.E-04 & Nannofossil ooze \\
\hline $16 \mathrm{H}-4,83-85$ & 146.55 & 163.46 & 4.36 & 1.65.E-04 & 4.74.E-07 & 7.00.E-04 & Diatom nannofossil ooze \\
\hline $16 \mathrm{H}-5,83-85$ & 148.06 & 164.97 & 5.35 & 2.66.E-04 & 8.52.E-07 & 9.61.E-04 & Nannofossil diatom ooze \\
\hline \multicolumn{8}{|l|}{ 303-U1308A- } \\
\hline $4 \mathrm{H}-3,58-60$ & 31.19 & 35.13 & 6.04 & $6.54 \mathrm{E}-05$ & $1.30 \mathrm{E}-07$ & 3.15.E-04 & Nannofossil ooze \\
\hline $4 \mathrm{H}-4,58-60$ & 32.69 & 36.63 & 6.43 & $1.33 \mathrm{E}-04$ & $2.38 \mathrm{E}-07$ & 4.81.E-04 & Nannofossil silty clay \\
\hline $4 \mathrm{H}-5,58-60$ & 34.19 & 38.13 & 5.67 & 7.71E-05 & $1.27 \mathrm{E}-07$ & 3.69.E-04 & Nannofossil ooze \\
\hline $5 \mathrm{H}-3,58-60$ & 40.67 & 45.51 & 7.43 & $5.06 \mathrm{E}-05$ & $2.64 \mathrm{E}-07$ & 2.35.E-04 & Silty clay \\
\hline $5 \mathrm{H}-4,58-60$ & 42.17 & 47.01 & 6.87 & $1.03 \mathrm{E}-04$ & $3.16 \mathrm{E}-07$ & 5.47.E-04 & Nannofossil silty clay \\
\hline $5 \mathrm{H}-5,58-60$ & 43.67 & 48.51 & 7.87 & $1.12 \mathrm{E}-04$ & $3.18 \mathrm{E}-07$ & 3.62.E-04 & Silty clay \\
\hline $5 \mathrm{H}-6,58-60$ & 45.17 & 50.01 & 7.04 & $2.08 \mathrm{E}-05$ & $1.77 \mathrm{E}-07$ & 3.65.E-04 & Nannofossil ooze \\
\hline $8 \mathrm{H}-4,58-60$ & 70.69 & 78.37 & 7.42 & $2.11 \mathrm{E}-05$ & $1.18 \mathrm{E}-07$ & 2.63.E-04 & Nannofossil ooze \\
\hline $8 \mathrm{H}-5,58-60$ & 72.19 & 79.87 & 9.00 & $1.47 \mathrm{E}-05$ & $2.99 \mathrm{E}-07$ & 2.18.E-04 & Silty clay \\
\hline
\end{tabular}


Table T1 (continued). (Continued on next page.)

\begin{tabular}{|c|c|c|c|c|c|c|c|}
\hline \multirow{2}{*}{$\begin{array}{l}\text { Core, section, } \\
\text { interval }(\mathrm{cm})\end{array}$} & \multicolumn{2}{|c|}{ Depth } & \multirow{2}{*}{$\begin{array}{l}\text { Sample } \\
\text { weight } \\
\text { (g) }\end{array}$} & \multirow{2}{*}{$\begin{array}{c}\mathrm{NRM} \\
\left(\mathrm{Am}^{2} / \mathrm{kg}\right)\end{array}$} & \multirow{2}{*}{$\begin{array}{c}\chi \\
\left(m^{3} / \mathrm{kg}\right)\end{array}$} & \multirow{2}{*}{$\begin{array}{c}\chi_{\text {ARM }} \\
\left(\mathrm{m}^{3} / \mathrm{kg}\right)\end{array}$} & \multirow[b]{2}{*}{ Lithology } \\
\hline & (mbsf) & (mcd) & & & & & \\
\hline $8 \mathrm{H}-6,58-60$ & 73.69 & 81.37 & 7.98 & $4.41 \mathrm{E}-05$ & $2.34 \mathrm{E}-07$ & 4.78.E-04 & Nannofossil ooze \\
\hline $10 \mathrm{H}-3,58-60$ & 88.19 & 97.41 & 7.95 & $7.73 \mathrm{E}-05$ & $3.03 \mathrm{E}-07$ & 4.92.E-04 & Nannofossil ooze \\
\hline $10 \mathrm{H}-4,58-60$ & 89.69 & 98.91 & 8.45 & $5.92 \mathrm{E}-05$ & $3.33 \mathrm{E}-07$ & 5.64.E-04 & Nannofossil ooze \\
\hline $10 \mathrm{H}-5,58-60$ & 91.19 & 100.41 & 8.58 & $7.78 \mathrm{E}-05$ & $3.46 \mathrm{E}-07$ & 6.13.E-04 & Nannofossil ooze \\
\hline $10 \mathrm{H}-6,58-60$ & 92.69 & 101.91 & 8.90 & $5.85 \mathrm{E}-05$ & $1.83 \mathrm{E}-07$ & 3.72.E-04 & Nannofossil ooze \\
\hline $12 \mathrm{H}-3,58-60$ & 107.19 & 117.10 & 9.55 & $1.94 \mathrm{E}-05$ & $7.32 \mathrm{E}-08$ & 1.88.E-04 & Nannofossil ooze \\
\hline $12 \mathrm{H}-4,58-60$ & 108.69 & 118.60 & 8.47 & $3.37 \mathrm{E}-05$ & $1.39 \mathrm{E}-07$ & 3.27.E-04 & Nannofossil ooze \\
\hline $12 \mathrm{H}-5,58-60$ & 110.19 & 120.10 & 7.32 & $5.60 \mathrm{E}-05$ & $2.13 \mathrm{E}-07$ & 4.43.E-04 & Nannofossil ooze \\
\hline $12 \mathrm{H}-6,58-60$ & 111.69 & 121.60 & 8.46 & $3.02 \mathrm{E}-05$ & $1.41 \mathrm{E}-07$ & 3.06.E-04 & Nannofossil ooze \\
\hline \multicolumn{8}{|l|}{ 303-U1308B- } \\
\hline $4 \mathrm{H}-3,58-60$ & 26.59 & 29.75 & 6.71 & $5.77 \mathrm{E}-05$ & $7.80 \mathrm{E}-08$ & 2.15.E-04 & Nannofossil ooze \\
\hline $4 \mathrm{H}-4,58-60$ & 28.09 & 31.25 & 7.87 & $6.58 \mathrm{E}-05$ & $2.06 \mathrm{E}-07$ & 3.73.E-04 & Nannofossil ooze \\
\hline $4 \mathrm{H}-5,58-60$ & 29.59 & 32.75 & 7.63 & $2.19 \mathrm{E}-05$ & $4.20 \mathrm{E}-08$ & 1.33.E-04 & Nannofossil ooze \\
\hline $4 \mathrm{H}-6,58-60$ & 31.09 & 34.25 & 7.57 & $3.20 \mathrm{E}-05$ & $1.15 \mathrm{E}-07$ & 2.36.E-04 & Nannofossil ooze \\
\hline $7 \mathrm{H}-4,58-60$ & 56.59 & 63.84 & 6.44 & $1.89 \mathrm{E}-05$ & $1.51 \mathrm{E}-07$ & 3.97.E-04 & Nannofossil ooze \\
\hline $7 \mathrm{H}-5,58-60$ & 58.09 & 65.34 & 8.88 & $2.74 \mathrm{E}-05$ & $3.22 \mathrm{E}-07$ & 4.71.E-04 & Nannofossil ooze \\
\hline $7 \mathrm{H}-6,58-60$ & 59.59 & 66.84 & 8.83 & $9.10 \mathrm{E}-05$ & $3.10 \mathrm{E}-07$ & 4.59.E-04 & Silty clay \\
\hline $8 \mathrm{H}-4,58-60$ & 66.09 & 72.90 & 8.37 & $2.63 \mathrm{E}-05$ & $1.58 \mathrm{E}-07$ & 3.06.E-04 & Nannofossil ooze \\
\hline $9 \mathrm{H}-2,58-60$ & 72.59 & 81.35 & 7.45 & $1.79 \mathrm{E}-05$ & $1.88 \mathrm{E}-07$ & 4.07.E-04 & Nannofossil ooze \\
\hline $9 \mathrm{H}-3,58-60$ & 74.09 & 82.85 & 6.56 & $3.82 \mathrm{E}-05$ & $5.14 \mathrm{E}-07$ & 8.17.E-04 & Nannofossil ooze \\
\hline $9 \mathrm{H}-4,58-60$ & 75.59 & 84.35 & 8.83 & $2.77 \mathrm{E}-05$ & $3.13 \mathrm{E}-07$ & 5.42.E-04 & Nannofossil ooze \\
\hline $9 \mathrm{H}-5,58-60$ & 77.09 & 85.85 & 8.90 & $6.01 \mathrm{E}-06$ & $3.11 \mathrm{E}-07$ & 4.94.E-04 & Silty clay nannofossil ooze \\
\hline $11 \mathrm{H}-2,58-60$ & 91.59 & 103.43 & 7.83 & $5.38 \mathrm{E}-05$ & $2.00 \mathrm{E}-07$ & 3.65.E-04 & Nannofossil ooze \\
\hline $11 \mathrm{H}-3,58-60$ & 93.09 & 104.93 & 8.69 & $1.64 \mathrm{E}-05$ & $1.29 \mathrm{E}-07$ & 2.79.E-04 & Nannofossil ooze \\
\hline $11 \mathrm{H}-4,58-60$ & 94.59 & 106.43 & 7.51 & $3.22 \mathrm{E}-05$ & $1.35 \mathrm{E}-07$ & 3.27.E-04 & Nannofossil ooze \\
\hline $11 \mathrm{H}-5,58-60$ & 96.09 & 107.93 & 8.42 & $2.11 \mathrm{E}-05$ & $3.64 \mathrm{E}-07$ & 4.53.E-04 & Nannofossil ooze \\
\hline $14 \mathrm{H}-3,58-60$ & 121.59 & 136.86 & 8.21 & $9.01 \mathrm{E}-05$ & $4.30 \mathrm{E}-07$ & 6.70.E-04 & Nannofossil clay \\
\hline $14 \mathrm{H}-4,58-60$ & 123.11 & 138.38 & 8.30 & $7.32 \mathrm{E}-05$ & $1.98 \mathrm{E}-07$ & 3.67.E-04 & Nannofossil ooze \\
\hline $14 \mathrm{H}-5,58-60$ & 124.61 & 139.88 & 6.50 & $2.49 \mathrm{E}-04$ & $6.36 \mathrm{E}-07$ & 9.97.E-04 & Nannofossil ooze \\
\hline \multicolumn{8}{|l|}{ 303-U1308C- } \\
\hline $1 \mathrm{H}-1,58-60$ & 0.59 & 0.59 & 5.59 & $9.40 \mathrm{E}-05$ & $2.46 \mathrm{E}-07$ & 4.42.E-04 & Silty clay \\
\hline $1 \mathrm{H}-2,58-60$ & 2.09 & 2.09 & 7.67 & $6.10 \mathrm{E}-05$ & $2.12 \mathrm{E}-07$ & 2.30.E-04 & Silty clay \\
\hline $2 \mathrm{H}-3,58-60$ & 8 & 9.89 & 7.09 & $8.46 \mathrm{E}-05$ & $2.65 \mathrm{E}-07$ & 3.69.E-04 & Nannofossil ooze \\
\hline $2 \mathrm{H}-4,58-60$ & 9.5 & 11.39 & 8.11 & $6.41 \mathrm{E}-05$ & $1.84 \mathrm{E}-07$ & 3.13.E-04 & Nannofossil ooze \\
\hline $2 \mathrm{H}-5,58-60$ & 11 & 12.89 & 8.30 & $6.25 \mathrm{E}-05$ & $1.61 \mathrm{E}-07$ & 2.57.E-04 & Nannofossil ooze \\
\hline $3 \mathrm{H}-3,58-60$ & 17.5 & 21.20 & 7.90 & $7.09 \mathrm{E}-05$ & $1.89 \mathrm{E}-07$ & 2.48.E-04 & Silty clay \\
\hline $3 \mathrm{H}-4,58-60$ & 19 & 22.70 & 8.26 & $3.88 \mathrm{E}-05$ & $7.53 \mathrm{E}-08$ & 1.65.E-04 & Nannofossil ooze \\
\hline $3 \mathrm{H}-5,58-60$ & 20.5 & 24.20 & 8.47 & $9.74 \mathrm{E}-06$ & $2.49 \mathrm{E}-08$ & 1.05.E-04 & Nannofossil ooze \\
\hline $3 \mathrm{H}-6,58-60$ & 22 & 25.70 & 6.55 & $4.08 \mathrm{E}-05$ & $1.09 \mathrm{E}-07$ & 2.15.E-04 & Nannofossil ooze \\
\hline $5 \mathrm{H}-2,58-60$ & 34.99 & 39.67 & 9.23 & $1.50 \mathrm{E}-05$ & $1.22 \mathrm{E}-07$ & 6.13.E-05 & Silty clay \\
\hline $5 \mathrm{H}-3,58-60$ & 36.49 & 41.17 & 7.56 & $8.13 \mathrm{E}-05$ & $2.21 \mathrm{E}-07$ & 2.97.E-04 & Nannofossil clay \\
\hline $5 \mathrm{H}-4,58-60$ & 37.99 & 42.67 & 9.37 & $1.77 \mathrm{E}-05$ & $1.11 \mathrm{E}-07$ & 1.19.E-04 & Silty clay \\
\hline $5 \mathrm{H}-5,58-60$ & 39.49 & 44.17 & 8.21 & $6.87 \mathrm{E}-05$ & $2.18 \mathrm{E}-07$ & 3.99.E-04 & Nannofossil ooze \\
\hline $6 \mathrm{H}-1,58-60$ & 42.99 & 49.64 & 7.69 & $2.21 \mathrm{E}-05$ & $7.30 \mathrm{E}-08$ & 1.73.E-04 & Nannofossil ooze \\
\hline $6 \mathrm{H}-2,58-60$ & 44.49 & 51.14 & 6.63 & $6.43 \mathrm{E}-05$ & $3.44 \mathrm{E}-07$ & 6.05.E-04 & Nannofossil silty clay \\
\hline $6 \mathrm{H}-3,58-60$ & 45.97 & 52.62 & 8.55 & $2.80 \mathrm{E}-05$ & $8.56 \mathrm{E}-08$ & 1.86.E-04 & Nannofossil ooze \\
\hline $6 \mathrm{H}-4,58-60$ & 47.46 & 54.11 & 7.30 & $6.69 \mathrm{E}-05$ & $2.78 \mathrm{E}-07$ & 5.10.E-04 & Nannofossil ooze \\
\hline $6 \mathrm{H}-5,58-60$ & 48.96 & 55.61 & 8.07 & $3.00 \mathrm{E}-05$ & $3.71 \mathrm{E}-07$ & 5.72.E-04 & Nannofossil ooze \\
\hline $8 \mathrm{H}-3,58-60$ & 64.99 & 74.23 & 6.78 & $4.71 \mathrm{E}-05$ & $2.99 \mathrm{E}-07$ & 5.27.E-04 & Diatom nannofossil ooze \\
\hline $8 \mathrm{H}-5,58-60$ & 67.99 & 77.23 & 6.71 & $5.80 \mathrm{E}-05$ & $4.18 \mathrm{E}-07$ & 7.14.E-04 & Diatom nannofossil ooze \\
\hline $13 \mathrm{H}-3,58-60$ & 112.49 & 126.90 & 6.08 & $5.34 \mathrm{E}-05$ & $6.48 \mathrm{E}-07$ & 1.02.E-03 & Nannofossil ooze \\
\hline $13 \mathrm{H}-4,58-60$ & 113.99 & 128.40 & 8.69 & $1.10 \mathrm{E}-05$ & $1.89 \mathrm{E}-07$ & 1.84.E-04 & Nannofossil ooze \\
\hline $13 \mathrm{H}-5,58-60$ & 115.49 & 129.90 & 7.39 & 4.33E-05 & $3.66 \mathrm{E}-07$ & 5.28.E-04 & Silty clay \\
\hline $13 \mathrm{H}-6,58-60$ & 116.99 & 131.40 & 7.40 & $5.79 \mathrm{E}-05$ & $2.67 \mathrm{E}-07$ & 4.54.E-04 & Nannofossil ooze \\
\hline $15 \mathrm{H}-3,58-60$ & 131.49 & 147.43 & 9.35 & $2.33 \mathrm{E}-05$ & $2.09 \mathrm{E}-07$ & 3.55.E-04 & Nannofossil ooze \\
\hline $15 \mathrm{H}-4,58-60$ & 132.99 & 148.93 & 9.37 & $3.13 \mathrm{E}-05$ & $2.56 \mathrm{E}-07$ & 3.48.E-04 & Nannofossil ooze \\
\hline $15 \mathrm{H}-5,58-60$ & 134.49 & 150.43 & 7.23 & $4.70 \mathrm{E}-05$ & $1.88 \mathrm{E}-07$ & 3.81.E-04 & Nannofossil ooze \\
\hline \multicolumn{8}{|l|}{ 303-U1308E- } \\
\hline $1 \mathrm{H}-2,58-60$ & 2.09 & 3.41 & 4.77 & $3.82 \mathrm{E}-05$ & 2.07E-07 & 3.32.E-04 & Nannofossil ooze \\
\hline $1 \mathrm{H}-3,58-60$ & 3.59 & 4.91 & 7.36 & $3.52 \mathrm{E}-05$ & $1.28 \mathrm{E}-07$ & 2.01.E-04 & Nannofossil silty clay \\
\hline $1 \mathrm{H}-4,58-60$ & 5.09 & 6.41 & 7.06 & $2.28 \mathrm{E}-05$ & 4.03E-08 & 1.51.E-04 & Nannofossil ooze \\
\hline $1 \mathrm{H}-5,58-60$ & 6.59 & 7.91 & 6.45 & $8.48 \mathrm{E}-06$ & $1.70 \mathrm{E}-08$ & 7.26.E-05 & Nannofossil ooze \\
\hline $1 \mathrm{H}-6,58-60$ & 8.09 & 9.41 & 7.40 & $9.16 \mathrm{E}-05$ & $2.38 \mathrm{E}-07$ & 2.70.E-04 & Nannofossil ooze \\
\hline $2 \mathrm{H}-2,58-60$ & 11.59 & 13.77 & 7.46 & $8.15 \mathrm{E}-05$ & $2.72 \mathrm{E}-07$ & 4.53.E-04 & Nannofossil silty clay \\
\hline $2 \mathrm{H}-3,58-60$ & 13.09 & 15.27 & 8.09 & $4.14 \mathrm{E}-05$ & $1.78 \mathrm{E}-07$ & 2.04.E-04 & Silty clay \\
\hline $2 \mathrm{H}-4,58-60$ & 14.59 & 16.77 & 9.09 & $2.85 \mathrm{E}-05$ & $1.39 \mathrm{E}-07$ & 1.92.E-04 & Nannofossil ooze \\
\hline $2 \mathrm{H}-5,58-60$ & 16.09 & 18.27 & 8.41 & $2.77 \mathrm{E}-05$ & $7.90 \mathrm{E}-08$ & 1.46.E-04 & Nannofossil ooze \\
\hline
\end{tabular}


Table T1 (continued).

\begin{tabular}{|c|c|c|c|c|c|c|c|}
\hline \multirow{2}{*}{$\begin{array}{l}\text { Core, section, } \\
\text { interval }(\mathrm{cm})\end{array}$} & \multicolumn{2}{|c|}{ Depth } & \multirow{2}{*}{$\begin{array}{c}\text { Sample } \\
\text { weight } \\
\text { (g) }\end{array}$} & \multirow{2}{*}{$\begin{array}{c}\text { NRM } \\
\left(\mathrm{Am}^{2} / \mathrm{kg}\right)\end{array}$} & \multirow{2}{*}{$\begin{array}{c}\chi \\
\left(m^{3} / k g\right)\end{array}$} & \multirow{2}{*}{$\begin{array}{c}\chi_{\text {ARM }} \\
\left(\mathrm{m}^{3} / \mathrm{kg}\right)\end{array}$} & \multirow[b]{2}{*}{ Lithology } \\
\hline & (mbsf) & $(\mathrm{mcd})$ & & & & & \\
\hline $2 \mathrm{H}-6,58-60$ & 17.59 & 19.77 & 7.31 & $5.43 \mathrm{E}-05$ & $1.77 \mathrm{E}-07$ & 3.69.E-04 & Nannofossil ooze \\
\hline $3 \mathrm{H}-2,58-60$ & 21.09 & 25.73 & 6.47 & $4.47 \mathrm{E}-05$ & $8.54 \mathrm{E}-08$ & 1.92.E-04 & Silty clay \\
\hline $3 \mathrm{H}-3,58-60$ & 22.59 & 27.23 & 8.25 & $6.88 \mathrm{E}-05$ & $3.10 \mathrm{E}-07$ & 2.98.E-04 & Nannofossil ooze \\
\hline $3 \mathrm{H}-4,58-60$ & 24.09 & 28.73 & 9.01 & $3.37 \mathrm{E}-05$ & $2.01 \mathrm{E}-07$ & 1.34.E-04 & Nannofossil ooze \\
\hline $9 \mathrm{H}-2,58-60$ & 81.09 & 86.56 & 6.74 & $4.35 \mathrm{E}-05$ & $4.14 \mathrm{E}-07$ & 6.48.E-04 & Silty clay \\
\hline $9 \mathrm{H}-4,58-60$ & 84.09 & 89.56 & 8.04 & $6.79 \mathrm{E}-05$ & $3.27 \mathrm{E}-07$ & 5.33.E-04 & Nannofossil ooze \\
\hline $12 \mathrm{H}-2,58-60$ & 108.59 & 121.66 & 8.52 & $3.32 \mathrm{E}-05$ & $1.72 \mathrm{E}-07$ & 3.62.E-04 & Nannofossil ooze \\
\hline $12 \mathrm{H}-3,58-60$ & 110.09 & 123.16 & 8.71 & $4.41 \mathrm{E}-05$ & $4.63 \mathrm{E}-07$ & 7.10.E-04 & Silty clay nannofossil ooze \\
\hline $12 \mathrm{H}-4,61-63$ & 111.62 & 124.69 & 8.15 & $9.94 \mathrm{E}-05$ & $4.99 \mathrm{E}-07$ & 7.24.E-04 & Silty clay nannofossil ooze \\
\hline $12 \mathrm{H}-5,58-60$ & 113.09 & 126.16 & 8.58 & $2.68 \mathrm{E}-05$ & $4.05 \mathrm{E}-07$ & 6.22.E-04 & Silty clay nannofossil ooze \\
\hline $12 \mathrm{H}-3,58-60$ & 119.59 & 134.72 & 7.44 & $5.81 \mathrm{E}-05$ & $3.50 \mathrm{E}-07$ & 5.62.E-04 & Silty clay nannofossil ooze \\
\hline \multicolumn{8}{|l|}{ 303-U1308F- } \\
\hline $6 \mathrm{H}-3,58-60$ & 50.52 & 56.96 & 7.38 & $1.29 \mathrm{E}-05$ & $2.70 \mathrm{E}-07$ & 2.70.E-04 & Silty clay \\
\hline $6 \mathrm{H}-4,58-60$ & 52.02 & 58.46 & 7.43 & $5.69 \mathrm{E}-05$ & $2.38 \mathrm{E}-07$ & 4.36.E-04 & Nannofossil ooze \\
\hline $6 \mathrm{H}-5,58-60$ & 53.52 & 59.96 & 7.40 & $1.82 \mathrm{E}-05$ & $3.88 \mathrm{E}-07$ & 4.45.E-04 & Nannofossil ooze \\
\hline $6 \mathrm{H}-6,58-60$ & 55.02 & 61.46 & 6.95 & $6.62 \mathrm{E}-05$ & $1.58 \mathrm{E}-07$ & 3.26.E-04 & Nannofossil ooze \\
\hline $7 \mathrm{H}-3,58-60$ & 60.59 & 69.32 & 8.26 & $9.19 \mathrm{E}-05$ & $2.10 \mathrm{E}-07$ & 4.11.E-04 & Nannofossil ooze \\
\hline $7 \mathrm{H}-4,58-60$ & 62.09 & 70.82 & 8.33 & $7.90 \mathrm{E}-05$ & $3.00 \mathrm{E}-07$ & 4.06.E-04 & Silty clay \\
\hline $7 \mathrm{H}-6,58-60$ & 65.09 & 73.82 & 7.82 & $1.23 \mathrm{E}-05$ & 3.37E-07 & 5.47.E-04 & Nannofossil ooze \\
\hline $9 \mathrm{H}-3,58-60$ & 79.59 & 91.29 & 8.62 & $1.98 \mathrm{E}-05$ & $2.88 \mathrm{E}-07$ & 3.10.E-04 & Nannofossil silty clay \\
\hline $9 \mathrm{H}-4,58-60$ & 81.09 & 92.79 & 7.85 & $4.91 \mathrm{E}-05$ & $1.72 \mathrm{E}-07$ & 3.46.E-04 & Nannofossil ooze \\
\hline $9 \mathrm{H}-5,58-60$ & 82.59 & 94.29 & 5.79 & $1.12 \mathrm{E}-04$ & $4.70 \mathrm{E}-07$ & 9.12.E-04 & Nannofossil ooze \\
\hline $9 \mathrm{H}-6,58-60$ & 84.09 & 95.79 & 5.08 & $1.26 \mathrm{E}-04$ & $2.55 \mathrm{E}-07$ & 5.93.E-04 & Nannofossil ooze \\
\hline $11 \mathrm{H}-1,58-60$ & 94.59 & 109.50 & 6.23 & $4.95 \mathrm{E}-05$ & $1.65 \mathrm{E}-07$ & 4.11.E-04 & Nannofossil ooze \\
\hline $11 \mathrm{H}-2,58-60$ & 96.09 & 111.00 & 5.48 & $8.85 \mathrm{E}-05$ & $3.13 \mathrm{E}-07$ & 6.63.E-04 & Nannofossil ooze \\
\hline $11 \mathrm{H}-3,58-60$ & 97.59 & 112.50 & 5.96 & $6.18 \mathrm{E}-05$ & $1.75 \mathrm{E}-07$ & 3.89.E-04 & Nannofossil silty clay \\
\hline $11 \mathrm{H}-4,58-60$ & 99.09 & 114.00 & 7.64 & $2.93 \mathrm{E}-06$ & $1.23 \mathrm{E}-07$ & 2.86.E-04 & Nannofossil ooze \\
\hline $11 \mathrm{H}-5,58-60$ & 100.59 & 115.50 & 5.84 & $1.31 \mathrm{E}-05$ & $4.36 \mathrm{E}-07$ & 8.00.E-04 & Nannofossil ooze \\
\hline $11 \mathrm{H}-6,58-60$ & 102.09 & 117.00 & 6.21 & $3.77 \mathrm{E}-05$ & $7.42 \mathrm{E}-08$ & 2.21.E-04 & Nannofossil ooze \\
\hline $13 \mathrm{H}-4,58-60$ & 118.12 & 133.36 & 6.46 & $1.49 \mathrm{E}-05$ & $3.60 \mathrm{E}-07$ & 6.08.E-04 & Nannofossil ooze \\
\hline $13 \mathrm{H}-5,58-60$ & 119.62 & 134.86 & 7.21 & $9.60 \mathrm{E}-05$ & $4.11 \mathrm{E}-07$ & 5.76.E-04 & Nannofossil ooze \\
\hline $13 \mathrm{H}-6,58-60$ & 121.12 & 136.36 & 6.64 & $1.27 \mathrm{E}-04$ & $3.00 \mathrm{E}-07$ & 5.64.E-04 & Nannofossil ooze \\
\hline $14 \mathrm{H}-2,58-60$ & 124.59 & 141.00 & 6.68 & $9.70 \mathrm{E}-05$ & $3.84 \mathrm{E}-07$ & 5.73.E-04 & Nannofossil ooze \\
\hline $14 \mathrm{H}-3,58-60$ & 126.09 & 142.50 & 6.61 & $2.14 \mathrm{E}-04$ & $4.11 \mathrm{E}-07$ & 6.21.E-04 & Nannofossil ooze \\
\hline $14 \mathrm{H}-6,58-60$ & 130.59 & 147.00 & 6.52 & $1.08 \mathrm{E}-05$ & $1.90 \mathrm{E}-07$ & 3.88.E-04 & Nannofossil ooze \\
\hline
\end{tabular}

Notes: NRM = natural remanent magnetization, $\chi=$ low-field magnetic susceptibility, $\chi_{\text {ARM }}=$ susceptibility of anhysteretic remanent magnetization (ARM). $\mathrm{mcd}=$ meters composite depth. 
Table T2. Magnetic hysteresis parameters and lithology, Sites U1302-U1304 and U1308. (See table notes.) (Continued on next page.)

\begin{tabular}{|c|c|c|c|c|c|c|c|c|c|c|c|}
\hline \multirow{2}{*}{$\begin{array}{l}\text { Core, section, } \\
\text { interval }(\mathrm{cm})\end{array}$} & \multicolumn{2}{|c|}{ Depth } & \multirow{2}{*}{$\begin{array}{l}\text { Sample } \\
\text { weight } \\
\text { (g) }\end{array}$} & \multirow{2}{*}{$\begin{array}{l}\mathrm{Hcr} \\
(\mathrm{mT})\end{array}$} & \multirow{2}{*}{$\begin{array}{c}\mathrm{Hc} \\
(\mathrm{mT})\end{array}$} & \multirow[b]{2}{*}{$\mathrm{Hcr} / \mathrm{Hc}$} & \multirow{2}{*}{$\begin{array}{c}\mathrm{Mr} \\
\left(\mathrm{Am}^{2} / \mathrm{kg}\right)\end{array}$} & \multirow{2}{*}{$\begin{array}{c}\mathrm{Ms} \\
\left(\mathrm{Am}^{2} / \mathrm{kg}\right)\end{array}$} & \multirow[b]{2}{*}{$\mathrm{Mr} / \mathrm{Ms}$} & & \\
\hline & (msbf) & $(\mathrm{mcd})$ & & & & & & & & $\left(\mathrm{m}^{3} / \mathrm{kg}\right)$ & Lithology \\
\hline 303-U1302B- & & & & & & & & & & & \\
\hline $1 \mathrm{H}-1,125-126$ & 1.25 & 1.25 & 0.93 & 32.6 & 9.24 & 3.53 & 1.07.E-02 & 1.15.E-01 & 0.09 & $3.50 \mathrm{E}-08$ & Silty clay \\
\hline $1 \mathrm{H}-6,2-3$ & 7.52 & 7.52 & 0.73 & 34.37 & 11.85 & 2.90 & 1.40.E-02 & 1.11.E-01 & 0.13 & $4.13 \mathrm{E}-08$ & Silty clay nannofossil ooze \\
\hline $2 \mathrm{H}-1,146-147$ & 11.16 & 11.16 & 0.49 & 35.16 & 13.93 & 2.52 & 9.50.E-03 & 5.61.E-02 & 0.17 & $2.03 \mathrm{E}-08$ & Silty clay \\
\hline $2 \mathrm{H}-6,2-3$ & 17.22 & 17.22 & 0.47 & 34.94 & 11.71 & 2.98 & 6.76.E-03 & 6.12.E-02 & 0.11 & $3.80 \mathrm{E}-08$ & Silty clay \\
\hline $3 \mathrm{H}-1,145-146$ & 20.65 & 20.65 & 0.57 & 33.84 & 13.52 & 2.50 & 1.28.E-02 & 8.29.E-02 & 0.15 & $5.16 \mathrm{E}-08$ & Silty clay nannofossil ooze \\
\hline $3 \mathrm{H}-6,3-4$ & 26.73 & 26.73 & 0.58 & 34.85 & 13.71 & 2.54 & 1.36.E-02 & 8.76.E-02 & 0.15 & $3.49 \mathrm{E}-08$ & Silty clay \\
\hline $4 \mathrm{H}-1,147-148$ & 30.17 & 30.17 & 0.72 & 32.35 & 10.10 & 3.20 & 1.13.E-02 & 1.09.E-01 & 0.10 & $3.43 \mathrm{E}-08$ & Silty clay \\
\hline $4 \mathrm{H}-6,2-3$ & 36.22 & 36.22 & 0.68 & 33.62 & 12.24 & 2.75 & 1.40.E-02 & 1.03.E-01 & 0.14 & $4.84 \mathrm{E}-08$ & Silty clay nannofossil \\
\hline $5 \mathrm{H}-1,8-9$ & 38.28 & 38.28 & 0.68 & 35.87 & 12.78 & 2.81 & 1.21.E-02 & 8.66.E-02 & 0.14 & $2.92 \mathrm{E}-08$ & Nannofossil ooze with silty clay \\
\hline $5 \mathrm{H}-6,8-9$ & 45.78 & 45.78 & 0.53 & 32.74 & 12.49 & 2.62 & 6.92.E-03 & 5.05.E-02 & 0.14 & $1.78 \mathrm{E}-08$ & Nannofossil ooze with silty clay \\
\hline $6 \mathrm{H}-1,128-129$ & 48.98 & 48.98 & 0.68 & 33.14 & 13.37 & 2.48 & 1.16.E-02 & 6.83.E-02 & 0.17 & $3.01 \mathrm{E}-08$ & Silty clay \\
\hline $6 \mathrm{H}-6,8-9$ & 55.28 & 55.28 & 0.61 & 34.57 & 11.76 & 2.94 & 8.50.E-03 & 7.13.E-02 & 0.12 & 4.47E-08 & Silty clay \\
\hline 7H-1, 123-124 & 58.43 & 58.43 & 0.41 & 32.81 & 11.58 & 2.83 & 9.09.E-03 & 7.27.E-02 & 0.12 & $1.60 \mathrm{E}-08$ & Clay with nannofossil ooze \\
\hline 7H-6, 8-9 & 64.78 & 64.78 & 0.53 & 33.16 & 12.02 & 2.76 & 1.01.E-02 & 7.34.E-02 & 0.14 & $1.93 \mathrm{E}-08$ & Clay with nannofossil ooze \\
\hline $8 \mathrm{H}-1,128-129$ & 67.98 & 67.98 & 0.72 & 31.38 & 10.93 & 2.87 & 1.01.E-02 & 8.55.E-02 & 0.12 & $3.72 \mathrm{E}-08$ & Silty clay \\
\hline $8 \mathrm{H}-6,8-9$ & 74.28 & 74.28 & 0.96 & 31.93 & 10.20 & 3.13 & 7.74.E-03 & 7.23.E-02 & 0.11 & $5.56 \mathrm{E}-08$ & Clay with nannofossil ooze \\
\hline $9 \mathrm{H}-1,118-119$ & 77.38 & 77.38 & 0.47 & 32.75 & 10.86 & 3.02 & 1.28.E-02 & 1.14.E-01 & 0.11 & $4.04 \mathrm{E}-08$ & Clay with nannofossil ooze \\
\hline $9 \mathrm{H}-6,18-19$ & 83.91 & 83.91 & 0.69 & 34.17 & 12.37 & 2.76 & 9.15.E-03 & 8.15.E-02 & 0.13 & $5.98 \mathrm{E}-08$ & Silty clay \\
\hline $10 \mathrm{H}-1,119-120$ & 86.89 & 86.89 & 0.68 & 31.52 & 9.75 & 3.23 & 5.58.E-03 & 5.84.E-02 & 0.10 & $2.79 \mathrm{E}-08$ & Silty clay \\
\hline 303-U1303A- & & & & & & & & & & & \\
\hline $1 \mathrm{H}-1,143-144$ & 1.43 & 8.89 & 0.51 & 35.38 & 13.75 & 2.57 & 1.61.E-02 & 9.59.E-02 & 0.17 & $4.55 \mathrm{E}-08$ & Clay with nannofossil ooze \\
\hline $1 \mathrm{H}-6,0-1$ & 7.5 & 14.96 & 0.75 & 33.73 & 10.05 & 3.36 & 9.57.E-03 & 9.64.E-02 & 0.10 & $3.74 \mathrm{E}-08$ & Clay with nannofossil ooze \\
\hline $2 \mathrm{H}-6,8-9$ & 15.98 & 23.79 & 0.77 & 33.74 & 11.26 & 3.00 & 7.74.E-03 & 6.78.E-02 & 0.11 & 4.17E-08 & Nannofossil clay \\
\hline $3 \mathrm{H}-1,143-144$ & 19.33 & 29.86 & 0.82 & 33.11 & 10.58 & 3.13 & 9.42.E-03 & 8.40.E-02 & 0.11 & $4.19 \mathrm{E}-08$ & Silty clay nannofossil ooze \\
\hline $3 \mathrm{H}-6,1-2$ & 25.41 & 35.94 & 0.68 & 33.57 & 12.24 & 2.74 & 1.26.E-02 & 9.01.E-02 & 0.14 & $2.94 \mathrm{E}-08$ & Silty clay nannofossil ooze \\
\hline $4 \mathrm{H}-1,120-121$ & 28.6 & 40.04 & 0.61 & 33.24 & 12.50 & 2.66 & 9.40.E-03 & 6.58.E-02 & 0.14 & $2.27 \mathrm{E}-08$ & Sand slit clay nannofossil \\
\hline $4 \mathrm{H}-2,147-248$ & 28.72 & 40.16 & 0.60 & 33.05 & 12.59 & 2.63 & 1.10.E-02 & 7.56.E-02 & 0.15 & $2.48 \mathrm{E}-08$ & Sand silt clay nannofossil \\
\hline $5 \mathrm{H}-1,147-248$ & 38.37 & 49.5 & 0.67 & 32.15 & 9.69 & 3.32 & 4.54.E-03 & 4.63.E-02 & 0.10 & $2.31 \mathrm{E}-08$ & Silty nannofossil ooze \\
\hline $5 \mathrm{H}-5,1-2$ & 42.91 & 54.04 & 0.62 & 33.4 & 13.00 & 2.57 & 1.44.E-02 & 9.47.E-02 & 0.15 & $3.53 \mathrm{E}-08$ & Clayey silt nannofossil ooze \\
\hline $6 \mathrm{H}-1,143-144$ & 47.83 & 59.32 & 0.52 & 25.29 & 3.52 & 7.19 & 6.36.E-03 & 2.09.E-04 & 0.03 & 7.70E-09 & Clayey nannofossil ooze \\
\hline $6 \mathrm{H}-6,1-2$ & 53.86 & 65.35 & 0.76 & 32.25 & 10.42 & 3.10 & 9.14.E-03 & 8.60.E-02 & 0.11 & $2.29 \mathrm{E}-08$ & Silty clay nannofossil ooze \\
\hline 7H-1, 118-119 & 57.08 & 68.59 & 0.64 & 31.28 & 7.30 & 4.28 & 4.18.E-03 & 6.36.E-02 & 0.07 & $2.91 \mathrm{E}-08$ & Clayey nannofossil ooze \\
\hline $8 \mathrm{H}-3,118-119$ & 69.58 & 78.9 & 0.74 & 30.68 & 5.54 & 5.54 & 5.28.E-03 & 8.27.E-03 & 0.05 & $1.78 \mathrm{E}-08$ & Sand silt clay nannofossil \\
\hline $9 \mathrm{H}-1,116-117$ & 76.06 & 83 & 0.69 & 27.32 & 4.98 & 5.48 & 8.72.E-03 & 1.88.E-04 & 0.05 & 2.37E-08 & Silty clay nannofossil ooze \\
\hline $10 \mathrm{H}-2,18-19$ & 86.08 & 92.81 & 0.81 & 30.9 & 9.04 & 3.42 & 1.03.E-02 & 1.19.E-01 & 0.09 & $3.86 \mathrm{E}-08$ & Clay with nannofossil ooze \\
\hline 303-U1304A- & & & & & & & & & & & \\
\hline $1 \mathrm{H}-1,108-109$ & 1.08 & 4.78 & 0.75 & 44.18 & 14.06 & 3.14 & 9.49.E-03 & 4.95.E-02 & 0.19 & $3.86 \mathrm{E}-08$ & Nannofossil ooze with clay \\
\hline $1 \mathrm{H}-6,18-19$ & 7.68 & 11.38 & 0.36 & 11.35 & 11.66 & 0.97 & 1.70.E-02 & 1.02.E-01 & 0.17 & $9.61 \mathrm{E}-09$ & Nannofossil ooze \\
\hline $2 \mathrm{H}-1,124-125$ & 10.74 & 15.76 & 0.55 & 33.59 & 11.59 & 2.90 & 1.55.E-02 & 9.26.E-02 & 0.17 & $1.30 \mathrm{E}-08$ & Diatom ooze \\
\hline $2 \mathrm{H}-6,18-19$ & 17.18 & 22.2 & 0.47 & 36.56 & 12.37 & 2.96 & 1.55.E-02 & 8.59.E-02 & 0.18 & $2.74 \mathrm{E}-08$ & Diatom nannofossil ooze \\
\hline $3 \mathrm{H}-1,141--142$ & 20.41 & 27.1 & 0.62 & 34.14 & 14.58 & 2.34 & 3.18.E-02 & 1.52.E-01 & 0.21 & $3.94 \mathrm{E}-08$ & Diatom nannofossil ooze \\
\hline $3 \mathrm{H}-6,8-9$ & 26.58 & 33.27 & 0.50 & 37.46 & 12.82 & 2.92 & 1.61.E-02 & 8.56.E-02 & 0.19 & $2.05 \mathrm{E}-08$ & Nannofossil ooze \\
\hline $4 \mathrm{H}-1,143-144$ & 29.93 & 37.13 & 0.69 & 32.22 & 8.63 & 3.73 & 9.04.E-03 & 9.27.E-02 & 0.10 & $2.40 \mathrm{E}-08$ & Silty clay nannofossil ooze \\
\hline $4 \mathrm{H}-6,1-2$ & 36.01 & 43.21 & 0.43 & 36.77 & 13.05 & 2.82 & 2.17.E-02 & 1.19.E-01 & 0.18 & $3.26 \mathrm{E}-08$ & Nannofossil ooze \\
\hline $5 \mathrm{H}-1,144-145$ & 39.44 & 47.7 & 0.48 & 30.93 & 8.52 & 3.63 & 7.81.E-03 & 6.41.E-02 & 0.12 & $1.40 \mathrm{E}-08$ & Diatom ooze \\
\hline $5 \mathrm{H}-6,1-2$ & 45.51 & 53.77 & 0.70 & 33.24 & 12.93 & 2.57 & 1.90.E-02 & 1.02.E-01 & 0.19 & $3.72 \mathrm{E}-08$ & Diatom nannofossil ooze \\
\hline $6 \mathrm{H}-1,143-144$ & 48.93 & 58.13 & 0.48 & 51.26 & 5.46 & 9.38 & 2.27.E-03 & 2.75.E-02 & 0.08 & $8.48 \mathrm{E}-09$ & Diatom ooze \\
\hline $6 \mathrm{H}-6,1-2$ & 55.01 & 64.21 & 0.89 & 36 & 8.79 & 4.10 & 3.52.E-03 & 2.36.E-02 & 0.15 & $2.50 \mathrm{E}-08$ & Diatom nannofossil ooze \\
\hline 7H-1, 149-150 & 58.49 & 70.25 & 0.55 & 33.4 & 10.60 & 3.15 & 1.00.E-02 & 6.18.E-02 & 0.16 & $8.81 \mathrm{E}-09$ & Nannofossil ooze \\
\hline $7 \mathrm{H}-6,1-1$ & 64.51 & 76.27 & 0.65 & 32.18 & 9.85 & 3.27 & 6.12.E-03 & 4.15.E-02 & 0.15 & $1.35 \mathrm{E}-08$ & Nannofossil ooze \\
\hline $8 \mathrm{H}-1,148-149$ & 67.98 & 81.08 & 0.45 & 33.49 & 11.54 & 2.90 & 1.76.E-02 & 1.03.E-01 & 0.17 & $1.30 \mathrm{E}-08$ & Nannofossil ooze \\
\hline $8 \mathrm{H}-6,1-2$ & 74.01 & 87.11 & 0.41 & 29.14 & 8.17 & 3.57 & 9.84.E-03 & 7.76.E-02 & 0.13 & $6.49 \mathrm{E}-09$ & Nannofossil ooze \\
\hline $9 \mathrm{H}-1,142-143$ & 77.42 & 91.57 & 0.45 & 28.33 & 5.84 & 4.86 & 3.60.E-03 & 3.83.E-02 & 0.09 & $6.27 \mathrm{E}-09$ & Diatom nannofossil ooze \\
\hline $9 \mathrm{H}-6,1-2$ & 83.54 & 97.69 & 0.37 & 20.29 & 2.48 & 8.17 & 1.16.E-03 & 2.92.E-02 & 0.04 & $6.92 \mathrm{E}-09$ & Nannofossil diatom ooze \\
\hline $10 \mathrm{H}-6,3-4$ & 93.11 & 107.77 & 0.45 & 30.27 & 9.08 & 3.33 & 1.01.E-02 & 7.34.E-02 & 0.14 & $1.15 \mathrm{E}-08$ & Nannofossil diatom ooze \\
\hline $11 \mathrm{H}-1,133-134$ & 96.33 & 112.25 & 0.31 & 31.2 & 9.47 & 3.29 & 1.38.E-02 & 9.57.E-02 & 0.14 & $4.22 \mathrm{E}-10$ & Nannofossil diatom ooze \\
\hline $11 \mathrm{H}-6,3-4$ & 102.53 & 118.45 & 0.40 & 36.81 & 13.62 & 2.70 & 2.90.E-02 & 1.47.E-01 & 0.20 & $2.75 \mathrm{E}-08$ & Clay \\
\hline $12 \mathrm{H}-1,141-142$ & 105.91 & 122.66 & 0.53 & 33.08 & 10.65 & 3.11 & 1.77.E-02 & 1.19.E-01 & 0.15 & $3.04 \mathrm{E}-08$ & Diatom silty clay \\
\hline $12 \mathrm{H}-6,3-4$ & 112.03 & 128.78 & 0.46 & 38.5 & 14.26 & 2.70 & 2.53.E-02 & 1.22.E-01 & 0.21 & $4.54 \mathrm{E}-08$ & Clay \\
\hline $13 \mathrm{H}-1,146-147$ & 115.46 & 133.58 & 0.36 & 38.21 & 13.73 & 2.78 & 3.04.E-02 & 1.52.E-01 & 0.20 & $3.06 \mathrm{E}-08$ & Nannofossil ooze \\
\hline $13 \mathrm{H}-6,3-4$ & 121.53 & 139.65 & 0.39 & 39.55 & 12.53 & 3.16 & 1.35.E-02 & 7.26.E-02 & 0.19 & $1.27 \mathrm{E}-08$ & Clay \\
\hline $14 \mathrm{H}-1,146-147$ & 124.96 & 144.56 & 0.58 & 41.87 & 15.56 & 2.69 & 2.13.E-02 & 9.40.E-02 & 0.23 & $1.89 \mathrm{E}-08$ & Nannofossil ooze \\
\hline $14 \mathrm{H}-6,3-4$ & 131.04 & 150.64 & 0.56 & 37.14 & 14.24 & 2.61 & 2.72.E-02 & 1.29.E-01 & 0.21 & $3.37 \mathrm{E}-08$ & Silty clay nannofossil ooze \\
\hline $15 \mathrm{H}-1,138-139$ & 134.38 & 154.34 & 0.47 & 35.11 & 13.47 & 2.61 & 3.10.E-02 & 1.67.E-01 & 0.19 & 4.33E-08 & Diatom nannofossil ooze \\
\hline $15 \mathrm{H}-6,1-2$ & 140.51 & 160.47 & 0.48 & 33.98 & 13.43 & 2.53 & 1.31.E-02 & 6.88.E-02 & 0.19 & $2.10 \mathrm{E}-08$ & Diatom nannofossil ooze \\
\hline
\end{tabular}


Table T2 (continued).

\begin{tabular}{|c|c|c|c|c|c|c|c|c|c|c|c|}
\hline \multirow{2}{*}{$\begin{array}{l}\text { Core, section, } \\
\text { interval }(\mathrm{cm})\end{array}$} & \multicolumn{2}{|c|}{ Depth } & \multirow{2}{*}{$\begin{array}{c}\text { Sample } \\
\text { weight } \\
\text { (g) }\end{array}$} & \multirow{2}{*}{$\begin{array}{l}\mathrm{Hcr} \\
(\mathrm{mT})\end{array}$} & \multirow{2}{*}{$\begin{array}{c}\mathrm{Hc} \\
(\mathrm{mT})\end{array}$} & \multirow[b]{2}{*}{$\mathrm{Hcr} / \mathrm{Hc}$} & \multirow{2}{*}{$\begin{array}{c}\mathrm{Mr} \\
\left(\mathrm{Am}^{2} / \mathrm{kg}\right)\end{array}$} & \multirow{2}{*}{$\begin{array}{c}\mathrm{Ms} \\
\left(\mathrm{Am}^{2} / \mathrm{kg}\right)\end{array}$} & \multirow[b]{2}{*}{$\mathrm{Mr} / \mathrm{Ms}$} & \multirow{2}{*}{$\begin{array}{l}\text { HFMS } \\
\left(\mathrm{m}^{3} / \mathrm{kg}\right)\end{array}$} & \multirow[b]{2}{*}{ Lithology } \\
\hline & (msbf) & $(\mathrm{mcd})$ & & & & & & & & & \\
\hline $16 \mathrm{H}-1,142-143$ & 143.92 & 164.94 & 0.52 & 41.15 & 18.26 & 2.25 & 2.08.E-02 & 8.14.E-02 & 0.25 & $2.70 \mathrm{E}-08$ & Diatom nannofossil ooze \\
\hline \multicolumn{12}{|l|}{ 303-U1308A- } \\
\hline $1 \mathrm{H}-1,138-139$ & 1.38 & 1.38 & 0.56 & 68.73 & 27.73 & 2.48 & 1.02.E-02 & 3.36.E-02 & 0.30 & $3.69 \mathrm{E}-08$ & Nannofossil ooze \\
\hline $1 \mathrm{H}-6,3-4$ & 7.53 & 7.53 & 0.40 & 37.18 & 14.00 & 2.66 & 1.24.E-03 & 9.12.E-03 & 0.14 & $7.82 \mathrm{E}-09$ & Nannofossil silty clay \\
\hline $2 \mathrm{H}-1,138-139$ & 9.98 & 10.96 & 0.38 & 35.03 & 13.39 & 2.62 & 2.65.E-03 & 1.72.E-02 & 0.15 & $6.61 \mathrm{E}-09$ & Silty clay \\
\hline $2 \mathrm{H}-6,3-4$ & 16.13 & 17.11 & 0.48 & 37.18 & 15.85 & 2.35 & 4.61.E-03 & 2.22.E-02 & 0.21 & $6.08 \mathrm{E}-09$ & Nannofossil ooze \\
\hline $3 \mathrm{H}-1,138-139$ & 19.48 & 20.9 & 0.57 & 33.95 & 11.59 & 2.93 & 7.78.E-03 & 6.09.E-02 & 0.13 & $2.79 \mathrm{E}-08$ & Nannofossil ooze \\
\hline $3 \mathrm{H}-6,3-4$ & 25.63 & 27.05 & 0.78 & 36.9 & 15.46 & 2.39 & 1.08.E-02 & 5.14.E-02 & 0.21 & $4.24 \mathrm{E}-08$ & Nannofossil silty clay \\
\hline $4 \mathrm{H}-1,138-139$ & 28.98 & 32.92 & 0.62 & 35.53 & 14.86 & 2.39 & 1.56.E-03 & 8.55.E-03 & 0.18 & $1.54 \mathrm{E}-09$ & Nannofossil ooze \\
\hline $4 \mathrm{H}-6,3-4$ & 35.13 & 39.07 & 0.81 & 36.36 & 16.11 & 2.26 & 1.15.E-02 & 4.89.E-02 & 0.23 & $3.26 \mathrm{E}-08$ & Silty clay \\
\hline $5 \mathrm{H}-1,144-145$ & 38.54 & 43.38 & 0.65 & 42.15 & 18.77 & 2.25 & 8.23.E-03 & 3.24.E-02 & 0.25 & $1.21 \mathrm{E}-08$ & Nannofossil ooze \\
\hline $6 \mathrm{H}-1,144-145$ & 48.04 & 54.13 & 0.54 & 37.76 & 16.61 & 2.27 & 6.15.E-03 & 2.71.E-02 & 0.23 & $1.19 \mathrm{E}-08$ & Nannofossil ooze \\
\hline $6 \mathrm{H}-6,1-2$ & 54.11 & 60.2 & 0.56 & 41.3 & 18.03 & 2.29 & 6.87.E-03 & 2.92.E-02 & 0.24 & $7.02 \mathrm{E}-09$ & Nannofossil ooze \\
\hline $7 \mathrm{H}-4,1-2$ & 60.61 & 68.52 & 0.50 & 40.72 & 17.95 & 2.27 & 8.06.E-03 & 3.35.E-02 & 0.24 & $7.66 \mathrm{E}-09$ & Nannofossil ooze \\
\hline $8 \mathrm{H}-1,147-248$ & 67.07 & 74.75 & 0.55 & 42.35 & 18.48 & 2.29 & 1.28.E-02 & 5.16.E-02 & 0.25 & $2.24 \mathrm{E}-08$ & Nannofossil ooze \\
\hline $8 \mathrm{H}-6,0-1$ & 73.1 & 80.78 & 0.40 & 43.74 & 19.23 & 2.27 & 1.78.E-02 & 6.81.E-02 & 0.26 & $1.62 \mathrm{E}-08$ & Nannofossil ooze \\
\hline $9 \mathrm{H}-6,0-1$ & 82.6 & 91.66 & 0.85 & 32.06 & 12.41 & 2.58 & 6.80.E-03 & 4.28.E-02 & 0.16 & $4.93 \mathrm{E}-08$ & Nannofossil silty clay \\
\hline $10 \mathrm{H}-1,144-145$ & 86.04 & 95.26 & 0.58 & 35.99 & 16.18 & 2.22 & 1.25.E-02 & 5.89.E-02 & 0.21 & $3.14 \mathrm{E}-08$ & Nannofossil ooze \\
\hline $10 \mathrm{H}-6,1-2$ & 92.11 & 101.33 & 0.58 & 39.74 & 17.91 & 2.22 & 6.32.E-03 & 2.60.E-02 & 0.24 & $1.08 \mathrm{E}-08$ & Nannofossil ooze \\
\hline $11 \mathrm{H}-2,3-4$ & 95.63 & 104.02 & 0.40 & 42.11 & 18.79 & 2.24 & 6.76.E-03 & 2.78.E-02 & 0.24 & $1.53 \mathrm{E}-09$ & Silty clay nannofossil ooze \\
\hline $11 \mathrm{H}-6,3-4$ & 101.63 & 110.02 & 0.42 & 41.13 & 18.37 & 2.24 & 1.19.E-02 & 4.78.E-02 & 0.25 & 8.67E-09 & Clay nannofossil ooze \\
\hline $12 \mathrm{H}-1,138-139$ & 104.98 & 114.89 & 0.55 & 36.75 & 16.45 & 2.23 & 1.54.E-02 & 6.42.E-02 & 0.24 & $2.18 \mathrm{E}-08$ & Nannofossil silty clay \\
\hline $12 \mathrm{H}-6,3-4$ & 111.13 & 121.04 & 0.42 & 35.67 & 15.25 & 2.34 & 3.18.E-03 & 1.65.E-02 & 0.05 & $9.45 \mathrm{E}-10$ & Nannofossil ooze \\
\hline $13 \mathrm{H}-1,138-139$ & 114.48 & 125.56 & 0.40 & 40.87 & 17.85 & 2.29 & 1.67.E-02 & 6.75.E-02 & 0.25 & $1.99 \mathrm{E}-08$ & Nannofossil ooze \\
\hline $13 \mathrm{H}-6,3-4$ & 120.58 & 131.66 & 0.48 & 39.52 & 17.74 & 2.23 & 1.38.E-02 & 5.58.E-02 & 0.25 & $1.90 \mathrm{E}-08$ & Nannofossil ooze \\
\hline $14 \mathrm{H}-1,138-139$ & 123.98 & 136.38 & 0.35 & 40.89 & 18.40 & 2.22 & 8.74.E-03 & 3.62.E-02 & 0.24 & $2.30 \mathrm{E}-09$ & Nannofossil ooze \\
\hline $14 \mathrm{H}-6,3-4$ & 130.13 & 142.53 & 0.63 & 41.65 & 18.93 & 2.20 & 1.26.E-02 & 4.80.E-02 & 0.26 & $2.15 \mathrm{E}-08$ & Nannofossil ooze \\
\hline $15 \mathrm{H}-6,3-4$ & 139.63 & 153.09 & 0.61 & 41.45 & 19.02 & 2.18 & 1.27.E-02 & 4.83.E-02 & 0.26 & $1.63 \mathrm{E}-08$ & Nannofossil ooze \\
\hline $16 \mathrm{H}-1,138-139$ & 142.98 & 158.88 & 0.60 & 32.01 & 11.18 & 2.86 & 3.20.E-03 & 2.67.E-02 & 0.12 & $2.12 \mathrm{E}-08$ & Nannofossil ooze \\
\hline
\end{tabular}

Notes: $\mathrm{Hcr}=$ remanent coercivity, $\mathrm{Hc}=$ coercivity, $\mathrm{Mr}=$ isothermal remanent magnetization, $\mathrm{Ms}=$ saturated magnetization, $\mathrm{HFMS}=$ high-field magnetic susceptibility. $\mathrm{mcd}=$ meters composite depth . 